$\operatorname{Argonne} \underset{\bigotimes}{\bigotimes}$

\title{
September 2007 Monitoring Results for Centralia, Kansas
}

\author{
Environmental Science Division
}




\begin{abstract}
About Argonne National Laboratory
Argonne is a U.S. Department of Energy laboratory managed by UChicago Argonne, LLC under contract DE-AC02-06CH11357. The Laboratory's main facility is outside Chicago, at 9700 South Cass Avenue, Argonne, Illinois 60439. For information about Argonne, see www.anl.gov.
\end{abstract}

\title{
Availability of This Report
}

This report is available, at no cost, at http://www.osti.gov/bridge. It is also available on paper to the U.S. Department of Energy and its contractors, for a processing fee, from:

U.S. Department of Energy

Office of Scientific and Technical Information

P.O. Box 62

Oak Ridge, TN 37831-0062

phone (865) 576-8401

fax (865) 576-5728

reports@adonis.osti.gov

\section{Disclaimer}

This report was prepared as an account of work sponsored by an agency of the United States Government. Reference herein to any specific commercial product, process, or service by trade name, trademark, manufacturer, or otherwise, does not necessarily constitute or imply its endorsement, recommendation, or favoring by the United States Government or any agency thereof. The views and opinions of document authors expressed herein do not necessarily state or reflect those of the United States Government or any agency thereof, Argonne National Laboratory, or UChicago Argonne, LLC. 


\section{September 2007 Monitoring Results for Centralia, Kansas}

by

Applied Geosciences and Environmental Management Section

Environmental Science Division, Argonne National Laboratory

January 2008

USDA 


\section{Contents}

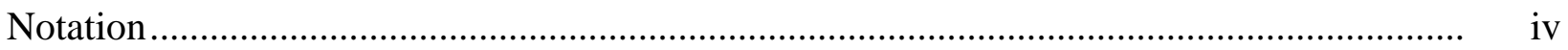

1 Introduction and Background................................................................................. 1-1

2 Sampling and Analysis Activities .......................................................................... 2-1

$2.1 \quad$ Measurement of Groundwater Levels ................................................................. 2-1

2.2 Monitoring Well and Piezometer Sampling and Analyses .................................... 2- 2-1

2.3 Handling and Disposal of Investigation-Derived Waste.......................................... 2-2

2.4 Quality Control for Sample Collection, Handling, and Analysis............................ 2-2

3 Results and Discussion........................................................................................ 3-1

3.1 Groundwater Level Data ............................................................................. 3-1

3.2 Groundwater Analysis Results .......................................................................... 3-1

3.2.1 Results of the Groundwater Analyses for VOCs ....................................... 3-1 3.2.2 Results of the Groundwater Analyses for Indicators of Possible
Contaminant Degradation ........................................................................... 3-2

4 Conclusions and Recommendations........................................................................... 4-1

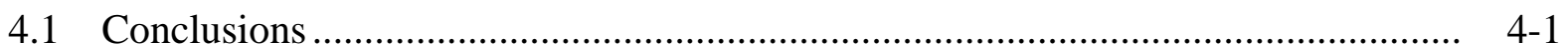

4.2 Recommendations .................................................................................. $4-1$

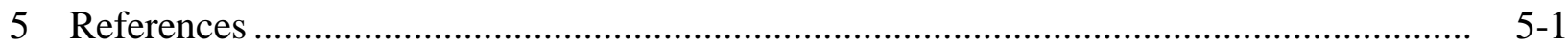

Appendix A: Sequence of Sampling Activities at Centralia, Kansas, in September 2007.................................................................................. A-1

Appendix B: Data Summary for Verification VOCs analyses by Envirosystems, Inc.......... B-1

\section{Tables}

3.1 Groundwater levels measured by hand at Centralia on July 27 and September 24-27, 2007

3.2 Analytical results from the AGEM Laboratory for volatile organic compounds in groundwater samples collected at Centralia, August 2004 to September 2007 . 
3.3 Field measurements for groundwater samples collected at Centralia, August 2004 to September 2007

3.4 Results of attenuation parameter analyses for groundwater samples collected at Centralia, August 2004 to September 2007

A.1 Sequence of sampling activities at Centralia in September 2007

\section{Figures}

1.1 Monitoring network at Centralia, as of September 2007

3.1 Potentiometric surface at Centralia, based on water levels measured manually on July 27, 2007

3.2 Carbon tetrachloride levels in groundwater at Centralia in March 2007, with the interpreted lateral extent of the contaminant at intervals during the period August 2004 to September 2007 


\section{Notation}

AGEM Applied Geosciences and Environmental Management

AMSL above mean sea level

BGL below ground level

${ }^{\circ} \mathrm{C} \quad$ degree(s) Celsius

CCC Commodity Credit Corporation

COC chain of custody

EDB ethylene dibromide

EPA U.S. Environmental Protection Agency

$\mathrm{ft} \quad$ foot (feet)

gal gallon(s)

$\mathrm{hr} \quad$ hour(s)

in. inch(es)

ISCR in situ chemical reduction

KDHE Kansas Department of Health and Environment

$\mu \mathrm{g} / \mathrm{L} \quad$ microgram(s) per liter

$\mu \mathrm{S} / \mathrm{cm} \quad$ microsiemen(s) per centimeter

$\mathrm{mg} / \mathrm{L} \quad$ milligram(s) per liter

$\mathrm{mV} \quad$ millivolt(s)

NAD North American Datum

NGVD National Geodetic Vertical Datum

nM nanomolar

ORP oxidation-reduction potential

TOC top of casing

TU tritium unit(s)

USDA U.S. Department of Agriculture

VOC volatile organic compound 


\section{September 2007 Monitoring Results for Centralia, Kansas}

\section{Introduction and Background}

In September 2005, periodic sampling of groundwater was initiated by the Commodity Credit Corporation of the U.S. Department of Agriculture (CCC/USDA) in the vicinity of a grain storage facility formerly operated by the CCC/USDA at Centralia, Kansas. The sampling at Centralia is being performed on behalf of the CCC/USDA by Argonne National Laboratory, in accord with a monitoring program approved by the Kansas Department of Health and Environment (KDHE). The objective is to monitor levels of carbon tetrachloride contamination identified in the groundwater at Centralia (Argonne 2003, 2004, 2005a). Under the KDHEapproved monitoring plan (Argonne 2005b), the groundwater is being sampled twice yearly (for a recommended period of two years) for analyses for volatile organic compounds (VOCs), as well as measurement of selected geochemical parameters to aid in the evaluation of possible natural contaminant degradation (reductive dechlorination) processes in the subsurface environment. The sampling is presently conducted in a network of 10 monitoring wells and 6 piezometers (Figure 1.1), at locations approved by the KDHE (Argonne 2006a).

The results of groundwater sampling and VOCs analyses in September-October 2005, March 2006, September 2006, and March 2007 were documented previously (Argonne 2006a,b, 2007a). The results have demonstrated the presence of carbon tetrachloride contamination, at levels exceeding the KDHE Tier 2 Risk-Based Screening Level of $5 \mu \mathrm{g} / \mathrm{L}$ for this compound, in a broad groundwater plume that has shown little movement.

This report presents the results of the groundwater sampling at Centralia in September 2007, performed in accord with the KDHE-approved monitoring plan (Argonne 2005b). The September 2007 sampling represents the fifth and final monitoring event performed under the recommended two-year monitoring program approved by the KDHE. 


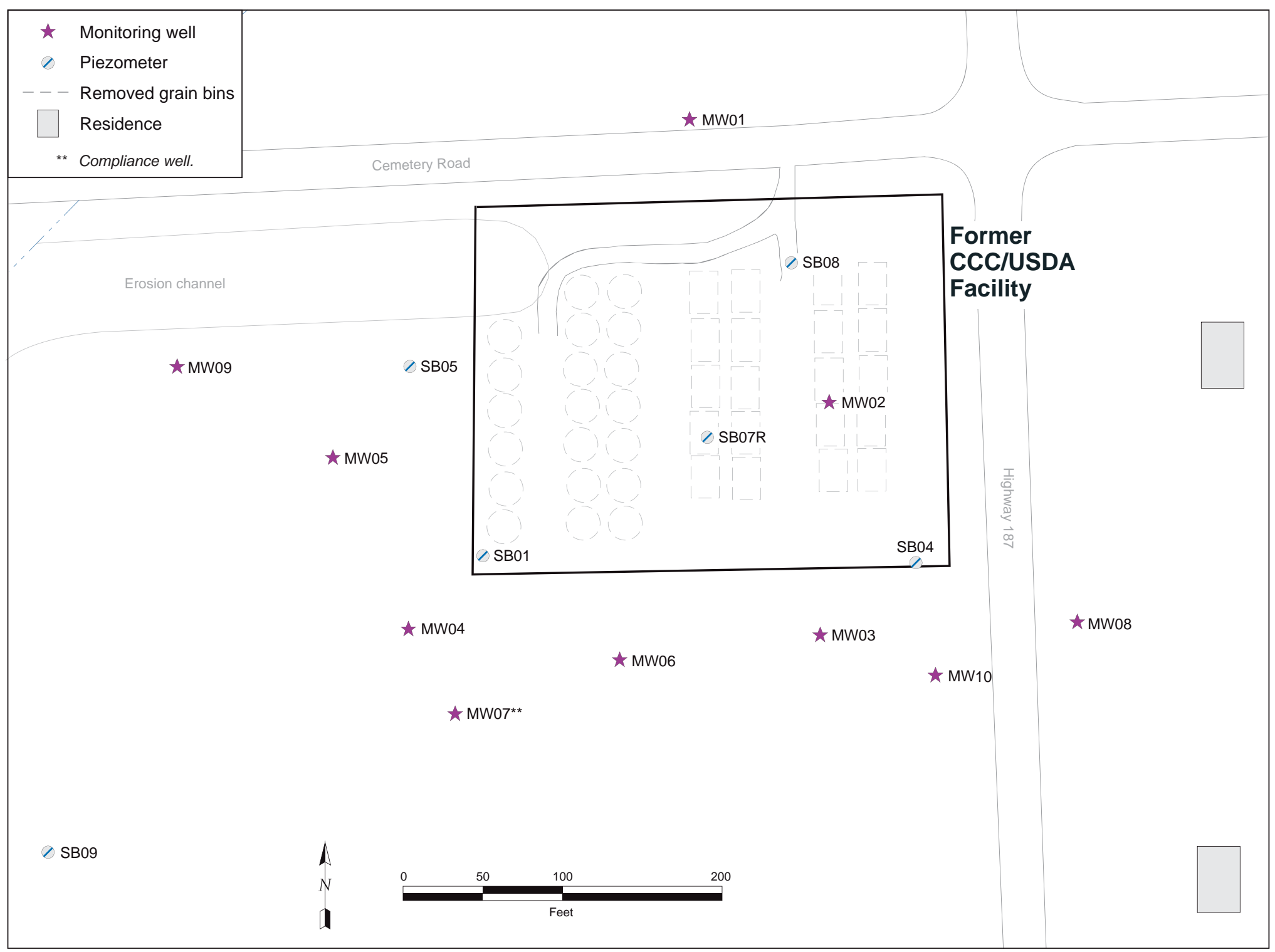

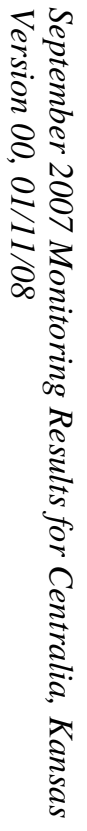

FIGURE 1.1 Monitoring network at Centralia, as of September 2007. 


\section{Sampling and Analysis Activities}

\subsection{Measurement of Groundwater Levels}

Monitoring wells MW01-MW10 and piezometers SB01, SB04, SB05, SB07R, SB08, and SB09 (Figure 1.1) were sampled on September 24-27, 2007. Before the wells were purged, a water level indicator was used to measure the depth to groundwater and the total depth of each well, to within $0.01 \mathrm{ft}$, from the top of the well casing.

Data recorders currently installed in MW01-MW06 are gathering long-term data on the groundwater elevation and gradient at Centralia. The data recorders in these wells were downloaded, and water levels were measured manually at all monitoring points, on July 27 , 2007.

The groundwater level data are discussed in Section 3.1.

\subsection{Monitoring Well and Piezometer Sampling and Analyses}

After measurement of water levels and dissolved oxygen levels, the monitoring points were purged of a minimum of three well volumes. Field measurements of temperature, $\mathrm{pH}$, and conductivity were taken during purging until the measurements stabilized. Field measurements of carbon dioxide, iron(II), and oxidation reduction potential (ORP) were made for the evaluation of possible biodegradation processes, as outlined in the monitoring plan (Argonne 2005b). All sampling and field analyses were performed in accord with procedures in the Master Work Plan (Argonne 2002). The sequence of activities during the September well sampling event is summarized in Appendix A.

Groundwater samples designated for VOCs analyses and selected geochemical analyses identified in the monitoring plan (Argonne 2005b) were collected in appropriate laboratory containers, labeled, packaged, and chilled to $4^{\circ} \mathrm{C}$ by placement in ice-filled coolers. The samples were shipped by an overnight delivery service to the Applied Geosciences and Environmental Management (AGEM) Laboratory at Argonne for VOCs analyses with U.S. Environmental Protection Agency (EPA) Method 524.2 (EPA 1995). Aliquots of selected samples (chosen in 
the field) were also shipped to Envirosystems, Inc., Columbia, Maryland, for verification VOCs analyses.

Samples designated for laboratory analyses of degradation parameters were collected and shipped to TestAmerica Laboratories (formerly Severn-Trent Laboratories), South Burlington, Vermont. The analyses included dissolved chloride, sulfate, nitrate, and phosphate concentrations by EPA Method 300; total alkalinity by EPA Method 310.1; nitrate-nitrite nitrogen by EPA Method 353.2; sulfide by EPA Method 376.2; total organic carbon by EPA Method 415.1; and dissolved metals (aluminum, calcium, iron, magnesium, manganese, phosphorus, potassium, silicon, sodium, and zinc) by EPA Method 6010 (EPA 1998a). Analyses for the natural attenuation indicators methane, ethane, and ethene were conducted with Method RSK-175 (Kampbell and Vandegrift 1998).

The analytical results are presented and discussed in Section 3.2.

\subsection{Handling and Disposal of Investigation-Derived Waste}

Purge water generated as potentially contaminated investigation-derived waste was containerized on-site. The accumulated purge water was sampled and analyzed by Pace Analytical Services, Inc., Lenexa, Kansas. Methods used were EPA Method 5030/8260 for VOCs, EPA Method 504.1 for ethylene dibromide (EDB), and EPA Method 300 for nitrate as nitrogen. Carbon tetrachloride was detected at $5.3 \mu \mathrm{g} / \mathrm{L}$, but EDB and other VOCs were not detected. Nitrate was present at $3.3 \mathrm{mg} / \mathrm{L}$. With the approval of KDHE, the water was disposed of at the Sabetha, Kansas, publicly owned treatment works on October 25, 2007 (Sabetha 2007).

\subsection{Quality Control for Sample Collection, Handling, and Analysis}

Quality assurance/quality control procedures followed during the September 2007 monitoring event are described in detail in the Master Work Plan (Argonne 2002). The results are summarized as follows:

- Sample collection and handling activities were monitored by the documentation of samples as they were collected and the use of chain-of- 
custody forms and custody seals to ensure sample integrity during handling and shipment.

- Samples designated for VOCs analyses were received with custody seals intact and at the appropriate preservation temperature. All samples were analyzed within the required holding times.

- Quality control samples (a field blank, an equipment rinsate, and trip blanks) were collected to monitor sample collection and handling activities. Method blanks were analyzed to monitor analytical methodologies. Carbon tetrachloride was detected in the rinsate sample associated with the sample from MW01 that was collected after sampling of contaminated well MW02. The trace concentration of carbon tetrachloride $(1.0 \mu \mathrm{g} / \mathrm{L})$ detected in the sample from MW01 is therefore discounted. Other quality control samples were free of carbon tetrachloride and chloroform contamination.

- Groundwater samples were analyzed for VOCs at the AGEM Laboratory with the purge-and-trap method on a gas chromatograph-mass spectrometer system. Calibration checks with each sample delivery group were required to be within $\pm 20 \%$ of the standard. Surrogate standard determinations performed on samples and blanks were within the specified range of $80-120 \%$ for all samples, in either the initial analysis or a successful reanalysis.

- In accordance with the procedures defined in the Master Work Plan (Argonne 2002), the analyses of water samples at the AGEM Laboratory were verified at a second laboratory. Two groundwater samples were analyzed according to the EPA's Contract Laboratory Program methodology by Envirosystems, Inc. For the samples from both monitoring well MW03 and piezometer SB07R, lower carbon tetrachloride concentrations were reported by Envirosytems than by the AGEM Laboratory. In contrast, for the chloroform results, agreement between the two laboratories was good. Low recovery was noted for some surrogate spikes in the Envirosystems analyses. Summary pages for the verification organic analyses are in Appendix B. 
- Samples shipped to TestAmerica Laboratories for attenuation parameter analyses were received with custody seals intact and at the appropriate preservation conditions. All samples were analyzed within the required holding times. (Although the analyses of some samples for nitrate with EPA Method 300 did occur beyond the 48-hr holding time limit, the primary analyses of preserved sample volumes for total nitrogen with EPA Method 353.2 were within the holding time limit.) Results were within the acceptable limits for laboratory quality control samples prepared and analyzed with the samples to evaluate accuracy and precision. 


\section{Results and Discussion}

\subsection{Groundwater Level Data}

Depths to groundwater were measured manually in all available monitoring wells on July 27, 2007, and September 24-27, 2007 (during sampling). The hand-measured water level data are in Table 3.1. The tables for Section 3 are grouped at the end of the section's text, before the figures.

The potentiometric surface at Centralia, interpreted from manual measurements on July 27, 2007, is depicted in Figure 3.1. The recent results are consistent with previous measurements (Figures 4.1-4.3 in Argonne 2006a and Figure 3.1 in Argonne 2007a), indicating an apparent groundwater flow direction toward the southwest across much of the former CCC/USDA facility. Like previous depictions of the potentiometric surface, Figure 3.1 indicates that groundwater flow is focused toward a localized low in the potentiometric surface, defined by the water level measurements at SB01, MW04, MW06, and MW07. Argonne's earlier investigations (Argonne 2003, 2004) suggested that the increased hydraulic gradients observed near these wells are a reflection of relatively low-permeability silts and clays that comprise the aquifer unit in this portion of the study area, in comparison to the more coarse-grained deposits identified in the northern and eastern portions of the site. The results of groundwater analyses at Centralia (discussed in Section 3.2) support an interpretation of slow groundwater flow (and carbon tetrachloride migration) to the south-southwest, in keeping with the observed water level patterns.

\subsection{Groundwater Analysis Results}

\subsubsection{Results of the Groundwater Analyses for VOCs}

The analytical data for VOCs in the groundwater samples collected in September 2007 are in Table 3.2, together with data for the previous sampling events conducted at Centralia since sampling of the monitoring well network began in 2004. The September 2007 data for carbon tetrachloride are illustrated in Figure 3.2, along with the lateral margins of the plume, as 
interpreted by Argonne on the basis of each of the groundwater sampling events summarized in Table 3.2.

Carbon tetrachloride was detected in September 2007 at 10 of the 16 monitoring locations on and downgradient from the former CCC/USDA facility (Figure 3.2), at concentrations ranging from $1.2 \mu \mathrm{g} / \mathrm{L}$ (at MW05) to a maximum of $1,138 \mu \mathrm{g} / \mathrm{L}$ (at MW02). Chloroform concentrations ranging from $<1 \mu \mathrm{g} / \mathrm{L}$ to $18 \mu \mathrm{g} / \mathrm{L}$ were detected at 6 of the 16 sampled locations (Table 3.2), in association with the more elevated carbon tetrachloride levels identified beneath the former CCC/USDA facility (at MW02, SB01, SB04, SB07R, and SB08) and immediately to the west (at SB05).

The present carbon tetrachloride concentrations are consistent with previous measurements. The data in Table 3.2 and Figure 3.2 continue to suggest longer-term trends of increasing carbon tetrachloride levels at monitoring points SB05, MW03, MW04, and MW07, along the western and southern margins of the groundwater plume and in the apparent direction of groundwater flow.

\subsubsection{Results of the Groundwater Analyses for Indicators of Possible Contaminant Degradation}

The results of field measurements and selected laboratory geochemical analyses on the groundwater samples are summarized in Tables 3.3 and 3.4. The reported parameters can be used to estimate whether the in situ conditions at Centralia are suitable for possible degradation of carbon tetrachloride by natural anaerobic (reductive dechlorination) processes, as outlined in regulatory guidance for the evaluation of these conditions (KDHE 2001; EPA 1998b).

The monitoring results to date give limited evidence for possible anaerobic biodegradation of carbon tetrachloride at Centralia. The persistent occurrence of the carbon tetrachloride breakdown product chloroform at relatively elevated concentrations at monitoring points SB01, SB05, and (particularly) MW02 during the March 2005 sampling event and each of the subsequent sampling events (Table 3.2 in the present report; see also Argonne 2005a, 2006a,b, 2007a) suggests that carbon tetrachloride is being degraded at these locations. 
TABLE 3.1 Groundwater levels measured by hand at Centralia on July 27 and September 24-27, 2007.

\begin{tabular}{|c|c|c|c|c|c|c|c|}
\hline \multirow[b]{3}{*}{ Well } & \multirow{2}{*}{\multicolumn{2}{|c|}{ Horizontal Location $^{\mathrm{a}}$ (ft) }} & \multirow{3}{*}{$\begin{array}{c}\text { Top of } \\
\text { Casing } \\
\text { Elevation }^{b} \\
\text { (ft AMSL) }^{c}\end{array}$} & \multicolumn{2}{|c|}{$\begin{array}{c}\text { Groundwater Level on } \\
\text { July } 27,2007\end{array}$} & \multicolumn{2}{|c|}{$\begin{array}{l}\text { Groundwater Level on } \\
\text { September } 24-27,2007\end{array}$} \\
\hline & & & & \multirow{2}{*}{$\begin{array}{l}\text { Depth } \\
(\text { ft TOC })^{d}\end{array}$} & \multirow{2}{*}{$\begin{array}{l}\text { Elevation } \\
\text { (ft AMSL) }\end{array}$} & \multirow{2}{*}{$\begin{array}{l}\text { Depth } \\
\text { (ft TOC) }\end{array}$} & \multirow{2}{*}{$\begin{array}{l}\text { Elevation } \\
\text { (ft AMSL) }\end{array}$} \\
\hline & Northing & Easting & & & & & \\
\hline MW01 & 515257.2 & 1839058.4 & 1329.30 & 13.42 & 1315.88 & 14.55 & 1314.75 \\
\hline MW02 & 515079.9 & 1839143.0 & 1334.82 & 22.51 & 1312.31 & 23.05 & 1311.77 \\
\hline MW03 & 514935.9 & 1839135.8 & 1334.70 & 22.37 & 1312.33 & 22.60 & 1312.10 \\
\hline MW04 & 514942.5 & 1838880.5 & 1322.71 & 26.09 & 1296.62 & 26.24 & 1296.47 \\
\hline MW05 & 515049.6 & 1838835.0 & 1318.11 & 11.17 & 1306.94 & 10.80 & 1307.31 \\
\hline MW06 & 514922.3 & 1839011.2 & 1329.82 & 38.37 & 1291.45 & 38.50 & 1291.32 \\
\hline MW07 & 514889.0 & 1838906.8 & 1324.83 & 29.90 & 1294.93 & 29.07 & 1295.76 \\
\hline MW08 & 514939.6 & 1839294.4 & 1332.41 & 20.15 & 1312.26 & 20.72 & 1311.69 \\
\hline MW09 & 515104.0 & 1838737.4 & 1310.49 & 3.51 & 1306.98 & 4.00 & 1306.49 \\
\hline MW10 & 514909.9 & 1839205.5 & 1334.56 & 22.28 & 1312.28 & 22.89 & 1311.67 \\
\hline SB01 & 514987.3 & 1838927.1 & 1325.16 & 19.52 & 1305.64 & 20.05 & 1305.11 \\
\hline SB04 & 514979.9 & 1839195.6 & 1335.73 & 23.42 & 1312.31 & 24.49 & 1311.24 \\
\hline SB07R & 515059.3 & 1839067.0 & 1331.71 & 19.89 & 1311.82 & 20.00 & 1311.71 \\
\hline SB08 & 515167.1 & 1839120.5 & 1332.56 & 20.25 & 1312.31 & 21.37 & 1311.19 \\
\hline SB09 & 514805.7 & 1838653.4 & 1311.04 & 7.02 & 1304.02 & 6.92 & 1304.12 \\
\hline
\end{tabular}

a Horizontal coordinates are target location centers. Northings and eastings are Kansas State Plane Coordinates. Horizontal datum is North American Datum (NAD) 83.

b Vertical datum is National Geodetic Vertical Datum (NGVD) 88.

c AMSL, above mean sea level.

d TOC, below top of casing.

Source of data: Schwab-Eaton, Manhattan, Kansas. 
TABLE 3.2 Analytical results from the AGEM Laboratory for volatile organic compounds in groundwater samples collected at Centralia, August 2004 to September 2007.

\begin{tabular}{|c|c|c|c|c|c|c|}
\hline \multirow[b]{2}{*}{ Well } & \multirow[b]{2}{*}{$\begin{array}{l}\text { Screen } \\
\text { Interval } \\
\text { (ft BGL) }\end{array}$} & \multirow[b]{2}{*}{ Sample } & \multirow[b]{2}{*}{$\begin{array}{l}\text { Sample } \\
\text { Date }\end{array}$} & \multicolumn{3}{|c|}{ Concentration ( $\mu \mathrm{g} / \mathrm{L})$} \\
\hline & & & & $\begin{array}{c}\text { Carbon } \\
\text { Tetrachloride }\end{array}$ & Chloroform & $\begin{array}{c}\text { Methylene } \\
\text { Chloride }\end{array}$ \\
\hline \multirow[t]{7}{*}{ MW01 } & $54.5-64.5$ & CNMW01-W-16158 & $8 / 24 / 04$ & $\mathrm{ND}^{\mathrm{a}}$ & ND & ND \\
\hline & & CNMW01-W-19276 & $9 / 10 / 05$ & ND & ND & ND \\
\hline & & CNMW01-W-16308 & $10 / 11 / 05$ & ND & ND & ND \\
\hline & & CNMW01-W-19890 & $3 / 15 / 06$ & ND & ND & ND \\
\hline & & CNMW01-W-22501 & $9 / 25 / 06$ & ND & ND & ND \\
\hline & & CNMW01-W-16326 & $3 / 29 / 07$ & ND & ND & ND \\
\hline & & CNMW01-W-16228 & $9 / 26 / 07$ & $1.0 \mathrm{R}^{\mathrm{b}}$ & ND & ND \\
\hline \multirow[t]{7}{*}{ MW02 } & $49.5-59.5$ & CNMW02-W-16159 & $8 / 26 / 04$ & 215 & 6.2 & ND \\
\hline & & CNMW02-W-19282 & $9 / 11 / 05$ & 776 & 33 & ND \\
\hline & & CNMW02-W-16309 & $10 / 12 / 05$ & 528 & 21 & ND \\
\hline & & CNMW02-W-19908 & $3 / 16 / 06$ & 847 & 21 & ND \\
\hline & & CNMW02-W-22508 & $9 / 26 / 06$ & 1233 & 25 & ND \\
\hline & & CNMW02-W-15489 & $3 / 26 / 07$ & 829 & 14 & ND \\
\hline & & CNMW02-W-16227 & $9 / 26 / 07$ & 1138 & 18 & ND \\
\hline \multirow[t]{7}{*}{ MW03 } & $50.5-60.5$ & CNMW03-W-16178 & $8 / 24 / 04$ & 1.2 & ND & ND \\
\hline & & CNMW03-W-19277 & $9 / 10 / 05$ & 1.6 & ND & ND \\
\hline & & CNMW03-W-16310 & $10 / 11 / 05$ & 1.8 & ND & ND \\
\hline & & CNMW03-W-19909 & $3 / 17 / 06$ & 2.6 & $0.2 \mathrm{Jc}$ & ND \\
\hline & & CNMW03-W-22513 & $9 / 26 / 06$ & 2.7 & ND & ND \\
\hline & & CNMW03-W-15494 & $3 / 27 / 07$ & 2.5 & ND & ND \\
\hline & & CNMW03-W-16223 & $9 / 25 / 07$ & 3.5 & ND & ND \\
\hline \multirow[t]{7}{*}{ MW04 } & $37.5-47.5$ & CNMW04-W-16180 & $8 / 24 / 04$ & ND & ND & ND \\
\hline & & CNMW04-W-19280 & $9 / 11 / 05$ & $0.9 \mathrm{~J}$ & ND & ND \\
\hline & & CNMW04-W-16311 & $10 / 11 / 05$ & $0.8 \mathrm{~J}$ & ND & ND \\
\hline & & CNMW04-W-19891 & $3 / 15 / 06$ & 1.3 & ND & ND \\
\hline & & CNMW04-W-22506 & $9 / 25 / 06$ & 1.4 & $0.1 \mathrm{~J}$ & ND \\
\hline & & CNMW04-W-16210 & $3 / 28 / 07$ & 2.1 & ND & ND \\
\hline & & CNMW04-W-16220 & $9 / 24 / 07$ & 2.0 & ND & ND \\
\hline \multirow[t]{7}{*}{ MW05 } & $34.5-44.5$ & CNMW05-W-16183 & $8 / 25 / 04$ & ND & ND & ND \\
\hline & & CNMW05-W-19279 & $9 / 10 / 05$ & 1.9 & ND & ND \\
\hline & & CNMW05-W-16312 & $10 / 11 / 05$ & 1.5 & ND & ND \\
\hline & & CNMW05-W-19976 & $3 / 15 / 06$ & 1.3 & ND & ND \\
\hline & & CNMW05-W-22505 & $9 / 25 / 06$ & 1.3 & ND & ND \\
\hline & & CNMW05-W-16213 & $3 / 28 / 07$ & $0.5 \mathrm{~J}$ & ND & ND \\
\hline & & CNMW05-W-16218 & $9 / 24 / 07$ & 1.2 & ND & ND \\
\hline \multirow[t]{7}{*}{ MW06 } & $46.5-56.5$ & CNMW06-W-16184 & $8 / 25 / 04$ & ND & ND & ND \\
\hline & & CNMW06-W-19278 & $9 / 10 / 05$ & ND & ND & ND \\
\hline & & CNMW06-W-16313 & $10 / 11 / 05$ & $0.3 \mathrm{~J}$ & ND & ND \\
\hline & & CNMW06-W-19889 & $3 / 15 / 06$ & $0.2 \mathrm{~J}$ & ND & ND \\
\hline & & CNMW06-W-22511 & $9 / 27 / 06$ & ND & ND & ND \\
\hline & & CNMW06-W-16208 & $3 / 27 / 07$ & ND & ND & ND \\
\hline & & CNMW06-W-16222 & $9 / 24 / 07$ & ND & ND & ND \\
\hline
\end{tabular}


TABLE 3.2 (Cont.)

\begin{tabular}{|c|c|c|c|c|c|c|}
\hline \multirow[b]{2}{*}{ Well } & \multirow[b]{2}{*}{$\begin{array}{l}\text { Screen } \\
\text { Interval } \\
\text { (ft BGL) }\end{array}$} & \multirow[b]{2}{*}{ Sample } & \multirow[b]{2}{*}{$\begin{array}{l}\text { Sample } \\
\text { Date }\end{array}$} & \multicolumn{3}{|c|}{ Concentration $(\mu \mathrm{g} / \mathrm{L})$} \\
\hline & & & & $\begin{array}{c}\text { Carbon } \\
\text { Tetrachloride }\end{array}$ & Chloroform & $\begin{array}{l}\text { Methylene } \\
\text { Chloride }\end{array}$ \\
\hline MW07 & $45-55$ & $\begin{array}{l}\text { CNMW07-W-19887 } \\
\text { CNMW07-W-22512 } \\
\text { CNMW07-W-15492 } \\
\text { CNMW07-W-16221 }\end{array}$ & $\begin{array}{l}3 / 14 / 06 \\
9 / 26 / 06 \\
3 / 26 / 07 \\
9 / 24 / 07\end{array}$ & $\begin{array}{l}0.4 \mathrm{~J} \\
1.1 \\
1.8 \\
2.4\end{array}$ & $\begin{array}{l}0.6 \mathrm{~J} \\
\text { ND } \\
\text { ND } \\
\text { ND }\end{array}$ & $\begin{array}{l}\text { ND } \\
\text { ND } \\
\text { ND } \\
\text { ND }\end{array}$ \\
\hline MW08 & $38-53$ & $\begin{array}{l}\text { CNMW08-W-19284 } \\
\text { CNMW08-W-22507 } \\
\text { CNMW08-W-15493 } \\
\text { CNMW08-W-16226 }\end{array}$ & $\begin{array}{l}3 / 14 / 06 \\
9 / 26 / 06 \\
3 / 27 / 07 \\
9 / 25 / 07\end{array}$ & $\begin{array}{l}\text { ND } \\
\text { ND } \\
\text { ND } \\
\text { ND }\end{array}$ & $\begin{array}{l}\text { ND } \\
\text { ND } \\
\text { ND } \\
\text { ND }\end{array}$ & $\begin{array}{l}\text { ND } \\
\text { ND } \\
\text { ND } \\
\text { ND }\end{array}$ \\
\hline MW09 & $25-35$ & $\begin{array}{l}\text { CNMW09-W-19285 } \\
\text { CNMW09-W-22504 } \\
\text { CNMW09-W-16209 } \\
\text { CNMW09-W-16219 }\end{array}$ & $\begin{array}{l}3 / 15 / 06 \\
9 / 25 / 06 \\
3 / 27 / 07 \\
9 / 24 / 07\end{array}$ & $\begin{array}{l}\text { ND } \\
\text { ND } \\
\text { ND } \\
\text { ND }\end{array}$ & $\begin{array}{l}\text { ND } \\
\text { ND } \\
\text { ND } \\
\text { ND }\end{array}$ & $\begin{array}{l}\text { ND } \\
\text { ND } \\
\text { ND } \\
\text { ND }\end{array}$ \\
\hline MW10 & $30-45$ & $\begin{array}{l}\text { CNMW10-W-19886 } \\
\text { CNMW10-W-22510 } \\
\text { CNMW10-W-16215 } \\
\text { CNMW10-W-16224 }\end{array}$ & $\begin{array}{l}3 / 14 / 06 \\
9 / 26 / 06 \\
3 / 28 / 07 \\
9 / 25 / 07\end{array}$ & $\begin{array}{l}\text { ND } \\
\text { ND } \\
\text { ND } \\
\text { ND }\end{array}$ & $\begin{array}{l}\text { ND } \\
\text { ND } \\
\text { ND } \\
\text { ND }\end{array}$ & $\begin{array}{l}\text { ND } \\
\text { ND } \\
\text { ND } \\
\text { ND }\end{array}$ \\
\hline SB01 & $40-50$ & $\begin{array}{l}\text { CNSB01-W-16188 } \\
\text { CNSB01-W-19274 } \\
\text { CNSB01-W-16314 } \\
\text { CNSB01-W-19979 } \\
\text { CNSB01-W-22516 } \\
\text { CNSB01-W-15491 } \\
\text { CNSB01-W-16232 }\end{array}$ & $\begin{array}{r}8 / 26 / 04 \\
9 / 9 / 05 \\
10 / 12 / 05 \\
3 / 17 / 06 \\
9 / 27 / 06 \\
3 / 27 / 07 \\
9 / 27 / 07\end{array}$ & $\begin{array}{l}186 \\
269 \\
288 \\
320 \\
267 \\
222 \\
283\end{array}$ & $\begin{array}{l}6.5 \\
6.8 \\
6.6 \\
5.7 \\
6.3 \\
4.9 \\
4.6\end{array}$ & $\begin{array}{l}\text { ND } \\
\text { ND } \\
\text { ND } \\
\text { ND } \\
\text { ND } \\
\text { ND } \\
\text { ND }\end{array}$ \\
\hline SB04 & $51-61$ & $\begin{array}{l}\text { CNSB04-W-16189 } \\
\text { CNSB04-W-19273 } \\
\text { CNSB04-W-16315 } \\
\text { CNSB04-W-19906 } \\
\text { CNSB04-W-22503 } \\
\text { CNSB04-W-16216 } \\
\text { CNSB04-W-16230 }\end{array}$ & $\begin{array}{r}8 / 26 / 04 \\
9 / 9 / 05 \\
10 / 12 / 05 \\
3 / 16 / 06 \\
9 / 25 / 06 \\
3 / 28 / 07 \\
9 / 26 / 07\end{array}$ & $\begin{array}{l}30 \\
47 \\
44 \\
51 \\
54 \\
44 \\
36\end{array}$ & $\begin{array}{l}N D \\
0.6 \mathrm{~J} \\
0.5 \mathrm{~J} \\
0.5 \mathrm{~J} \\
0.7 \mathrm{~J} \\
0.5 \mathrm{~J} \\
0.4 \mathrm{~J}\end{array}$ & $\begin{array}{l}N D \\
N D \\
N D \\
0.4 \mathrm{JB}^{\mathrm{d}} \\
\mathrm{ND} \\
\mathrm{ND} \\
\mathrm{ND}\end{array}$ \\
\hline SB05 & $32-42$ & $\begin{array}{l}\text { CNSB05-W-16190 } \\
\text { CNSB05-W-19275 } \\
\text { CNSB05-W-16323 } \\
\text { CNSB05-W-19904 } \\
\text { CNSB05-W-19940 } \\
\text { CNSB05-W-16212 } \\
\text { CNSB05-W-16233 }\end{array}$ & $\begin{array}{r}8 / 26 / 04 \\
9 / 9 / 05 \\
10 / 12 / 05 \\
3 / 17 / 06 \\
9 / 27 / 06 \\
3 / 28 / 07 \\
9 / 26 / 07\end{array}$ & $\begin{array}{c}59 \\
77 \\
54 \\
104 \\
139 \\
138 \\
221\end{array}$ & $\begin{array}{l}5.5 \\
7.2 \\
5.5 \\
7.2 \\
12 \\
12 \\
16\end{array}$ & $\begin{array}{l}\text { ND } \\
\text { ND } \\
\text { ND } \\
\text { ND } \\
\text { ND } \\
\text { ND } \\
\text { ND }\end{array}$ \\
\hline SB07R & $45-60$ & $\begin{array}{l}\text { CNSB07R-W-19978 } \\
\text { CNSB07R-W-19924 } \\
\text { CNSB07R-W-15490 } \\
\text { CNSB07R-W-16225 }\end{array}$ & $\begin{array}{l}3 / 15 / 06 \\
9 / 26 / 06 \\
3 / 26 / 07 \\
9 / 25 / 07\end{array}$ & $\begin{array}{l}41 \\
30 \\
30 \\
50\end{array}$ & $\begin{array}{l}2.7 \\
1.7 \\
1.7 \\
2.4\end{array}$ & $\begin{array}{l}\text { ND } \\
\text { ND } \\
\text { ND } \\
\text { ND }\end{array}$ \\
\hline
\end{tabular}


TABLE 3.2 (Cont.)

\begin{tabular}{|c|c|c|c|c|c|c|}
\hline \multirow[b]{2}{*}{ Well } & \multirow[b]{2}{*}{$\begin{array}{l}\text { Screen } \\
\text { Interval } \\
\text { (ft BGL) }\end{array}$} & \multirow[b]{2}{*}{ Sample } & \multirow[b]{2}{*}{$\begin{array}{l}\text { Sample } \\
\text { Date }\end{array}$} & \multicolumn{3}{|c|}{ Concentration $(\mu \mathrm{g} / \mathrm{L})$} \\
\hline & & & & $\begin{array}{c}\text { Carbon } \\
\text { Tetrachloride }\end{array}$ & Chloroform & $\begin{array}{c}\text { Methylene } \\
\text { Chloride }\end{array}$ \\
\hline \multirow[t]{7}{*}{ SB08 } & $52-62$ & CNSB08-W-16192 & 8/26/04 & 79 & 3.1 & ND \\
\hline & & CNSB08-W-19272 & $9 / 8 / 05$ & 80 & 2.6 & ND \\
\hline & & CNSB08-W-16317 & $10 / 12 / 05$ & 77 & 2.8 & ND \\
\hline & & CNSB08-W-19903 & $3 / 17 / 06$ & 91 & 2.7 & ND \\
\hline & & CNSB08-W-22500 & $9 / 21 / 06$ & 53 & 1.6 & ND \\
\hline & & CNSB08-W-16214 & $3 / 28 / 07$ & 64 & 2.0 & ND \\
\hline & & CNSB08-W-16229 & $9 / 26 / 07$ & 68 & 1.8 & ND \\
\hline \multirow[t]{7}{*}{ SB09 } & $32-42$ & CNSB09-W-16193 & $8 / 26 / 04$ & ND & ND & ND \\
\hline & & CNSB09-W-19281 & $9 / 11 / 05$ & ND & ND & ND \\
\hline & & CNSB09-W-16318 & $10 / 11 / 05$ & ND & ND & ND \\
\hline & & CNSB09-W-19902 & $3 / 17 / 06$ & ND & ND & ND \\
\hline & & CNSB09-W-22502 & $9 / 25 / 06$ & ND & ND & ND \\
\hline & & CNSB09-W-16211 & $3 / 28 / 07$ & ND & ND & ND \\
\hline & & CNSB09-W-16231 & $9 / 26 / 07$ & ND & ND & ND \\
\hline
\end{tabular}

a ND, not detected at an instrument detection limit of $0.1 \mu \mathrm{g} / \mathrm{L}$.

${ }^{b}$ Qualifier R indicates that the contaminant was present in the associated equipment rinsate.

${ }^{\mathrm{C}}$ Qualifier $\mathrm{J}$ indicates an estimated concentration below the method quantitation limit of $1.0 \mu \mathrm{g} / \mathrm{L}$.

d Qualifier B indicates that the contaminant was present in the associated method blank. 
TABLE 3.3 Field measurements for groundwater samples collected at Centralia, August 2004 to September 2007.

\begin{tabular}{|c|c|c|c|c|c|c|c|c|c|}
\hline \multirow[b]{2}{*}{ Location } & \multirow{2}{*}{$\begin{array}{l}\text { Screen } \\
\text { Interval } \\
\text { (ft BGL) }\end{array}$} & \multirow[b]{2}{*}{$\begin{array}{l}\text { Sample } \\
\text { Date }\end{array}$} & \multirow[b]{2}{*}{$\begin{array}{c}\text { Temperature } \\
\left({ }^{\circ} \mathrm{C}\right)\end{array}$} & \multirow[b]{2}{*}{$\mathrm{pH}$} & \multirow[b]{2}{*}{$\begin{array}{l}\text { Conductivity } \\
(\mu \mathrm{S} / \mathrm{cm})\end{array}$} & \multicolumn{3}{|c|}{ Concentration (mg/L) } & \multirow[b]{2}{*}{$\begin{array}{l}\text { ORP } \\
(\mathrm{mV})\end{array}$} \\
\hline & & & & & & $\begin{array}{l}\text { Dissolved } \\
\text { Oxygen }\end{array}$ & $\begin{array}{l}\text { Carbon } \\
\text { Dioxide }\end{array}$ & Iron(II) & \\
\hline \multirow[t]{6}{*}{ MW01 } & $54.5-64.5$ & $\begin{array}{l}8 / 24 / 04 \\
9 / 10 / 05\end{array}$ & $\begin{array}{l}16.3 \\
16.3\end{array}$ & $\begin{array}{l}7.39 \\
7.26\end{array}$ & $\begin{array}{l}652 \\
599\end{array}$ & $\begin{array}{l}0.06 \\
6.31\end{array}$ & $\begin{array}{l}25 \\
-a\end{array}$ & $\begin{array}{l}0 \\
0\end{array}$ & $\begin{array}{l}230 \\
104\end{array}$ \\
\hline & & $10 / 11 / 05$ & 16.4 & 6.45 & 634 & - & - & - & - \\
\hline & & $3 / 15 / 06$ & 14.3 & 7.56 & 621 & 9.33 & 30 & 0.04 & 297 \\
\hline & & $9 / 25 / 06$ & 13.3 & 7.01 & 782 & 6.82 & 50 & 0.31 & 92 \\
\hline & & $3 / 29 / 07$ & 16.5 & 6.54 & 629 & 4.39 & - & 0 & 174 \\
\hline & & $9 / 26 / 07$ & 17.8 & 7.06 & 630 & 0.89 & 35 & 0.09 & 146 \\
\hline \multirow[t]{7}{*}{ MW02 } & $49.5-59.5$ & $8 / 26 / 04$ & 14.4 & 7.31 & 729 & 0.16 & 20 & 0.12 & 235 \\
\hline & & $9 / 11 / 05$ & 15.3 & 7.02 & 739 & 1.28 & - & - & - \\
\hline & & $10 / 12 / 05$ & 14.8 & 6.60 & 766 & - & - & - & - \\
\hline & & $3 / 16 / 06$ & 14.2 & 6.78 & 759 & 1.24 & - & 0 & 295 \\
\hline & & $9 / 26 / 06$ & 13.2 & 6.98 & 957 & 3.05 & 40 & 0.06 & 67 \\
\hline & & $3 / 26 / 07$ & 15.7 & 6.39 & 739 & 2.29 & 50 & - & 67 \\
\hline & & $9 / 26 / 07$ & 15.4 & 7.04 & 763 & 3.39 & 25 & 0 & 156 \\
\hline \multirow[t]{7}{*}{ MW03 } & $50.5-60.5$ & $8 / 24 / 04$ & 13.1 & 7.28 & 783 & 0.10 & 55 & 0.21 & 230 \\
\hline & & $9 / 10 / 05$ & 15.1 & 7.05 & 715 & 10.42 & 65 & 0 & 142 \\
\hline & & $10 / 11 / 05$ & 16.3 & 6.46 & 765 & - & - & - & - \\
\hline & & $3 / 17 / 06$ & 13.8 & 6.75 & 753 & 9.39 & 77 & 0 & 290 \\
\hline & & 9/26/06 & 13.2 & 6.92 & 960 & 11.57 & 45 & 0.08 & 251 \\
\hline & & $3 / 27 / 07$ & 15.3 & 6.40 & 774 & 7.73 & 25 & - & 268 \\
\hline & & $9 / 25 / 07$ & 14.3 & 6.97 & 738 & 8.44 & 30 & 0 & 162 \\
\hline \multirow[t]{7}{*}{ MW04 } & $37.5-47.5$ & $8 / 24 / 04$ & 16.2 & 7.39 & 717 & 0.11 & 40 & 0.04 & 210 \\
\hline & & $9 / 11 / 05$ & 15.4 & 7.18 & 665 & 8.43 & 60 & 0 & 226 \\
\hline & & $10 / 11 / 05$ & 14.4 & 7.14 & 811 & - & - & - & - \\
\hline & & $3 / 15 / 06$ & 13.5 & 7.78 & 675 & 6.82 & 55 & 0.06 & 283 \\
\hline & & $9 / 25 / 06$ & - & 7.02 & 613 & 9.13 & 40 & 0.19 & 46 \\
\hline & & $3 / 28 / 07$ & 15.4 & 6.47 & 678 & 5.46 & - & 0 & 197 \\
\hline & & $9 / 24 / 07$ & 17.4 & 7.10 & 667 & 6.94 & 35 & 0.24 & 261 \\
\hline \multirow[t]{7}{*}{ MW05 } & $34.5-44.5$ & 8/25/04 & 14.3 & 7.14 & 613 & 0.08 & 25 & 0.06 & 215 \\
\hline & & 9/10/05 & 14.2 & 6.80 & 620 & 1.40 & 110 & 0 & 160 \\
\hline & & $10 / 11 / 05$ & 14.8 & 6.35 & 610 & - & - & - & - \\
\hline & & $3 / 15 / 06$ & 14.3 & 6.90 & 701 & 0.90 & 30 & 0.06 & 156 \\
\hline & & $9 / 25 / 06$ & 13.6 & 6.95 & 768 & 0.09 & 50 & 0.02 & 55 \\
\hline & & $3 / 28 / 07$ & 14.4 & 6.44 & 573 & 4.53 & 35 & 0 & 295 \\
\hline & & $9 / 24 / 07$ & 15.8 & 7.06 & 368 & 3.09 & 45 & 0 & 182 \\
\hline \multirow[t]{7}{*}{ MW06 } & $46.5-56.5$ & 8/25/04 & 15.9 & 7.50 & 637 & 0.05 & 15 & 0 & 215 \\
\hline & & 9/10/05 & 14.6 & 7.23 & 659 & 0.04 & 60 & 0 & 41 \\
\hline & & $10 / 11 / 05$ & 15.8 & 6.99 & 638 & - & - & - & - \\
\hline & & $3 / 15 / 06$ & 14.1 & 7.38 & 630 & 9.87 & 35 & 0.02 & 263 \\
\hline & & 9/27/06 & 13.1 & 6.16 & 652 & 0.05 & 45 & 1.12 & 63 \\
\hline & & $3 / 27 / 07$ & 19.0 & 6.42 & 466 & 0.11 & 20 & 0 & 13 \\
\hline & & $9 / 24 / 07$ & 16.8 & 7.11 & 463 & 8.00 & 25 & 0.41 & 191 \\
\hline
\end{tabular}


TABLE 3.3 (Cont.)

\begin{tabular}{|c|c|c|c|c|c|c|c|c|c|}
\hline \multirow[b]{2}{*}{ Location } & \multirow{2}{*}{$\begin{array}{l}\text { Screen } \\
\text { Interval } \\
\text { (ft BGL) }\end{array}$} & \multirow[b]{2}{*}{$\begin{array}{l}\text { Sample } \\
\text { Date }\end{array}$} & \multirow[b]{2}{*}{$\begin{array}{c}\text { Temperature } \\
\left({ }^{\circ} \mathrm{C}\right)\end{array}$} & \multirow[b]{2}{*}{$\mathrm{pH}$} & \multirow[b]{2}{*}{$\begin{array}{l}\text { Conductivity } \\
\qquad(\mu \mathrm{S} / \mathrm{cm})\end{array}$} & \multicolumn{3}{|c|}{ Concentration (mg/L) } & \multirow[b]{2}{*}{$\begin{array}{l}\text { ORP } \\
(\mathrm{mV})\end{array}$} \\
\hline & & & & & & $\begin{array}{l}\text { Dissolved } \\
\text { Oxygen }\end{array}$ & $\begin{array}{l}\text { Carbon } \\
\text { Dioxide }\end{array}$ & Iron(II) & \\
\hline MW07 & $45-55$ & $\begin{array}{l}3 / 14 / 06 \\
9 / 26 / 06 \\
3 / 26 / 07 \\
9 / 24 / 07\end{array}$ & $\begin{array}{l}14.7 \\
13.1 \\
15.8 \\
19.0\end{array}$ & $\begin{array}{l}6.61 \\
7.23 \\
6.50 \\
7.18\end{array}$ & $\begin{array}{l}709 \\
642 \\
642 \\
609\end{array}$ & $\begin{array}{l}0.34 \\
2.91 \\
1.87 \\
9.05\end{array}$ & $\begin{array}{l}- \\
50 \\
30 \\
60\end{array}$ & $\begin{array}{c}0.03 \\
0 \\
0 \\
0.18\end{array}$ & $\begin{array}{r}143 \\
- \\
261 \\
190\end{array}$ \\
\hline MW08 & $38-53$ & $\begin{array}{l}3 / 14 / 06 \\
9 / 26 / 06 \\
3 / 27 / 07 \\
9 / 25 / 07\end{array}$ & $\begin{array}{l}13.5 \\
13.3 \\
15.8 \\
15.8\end{array}$ & $\begin{array}{l}6.35 \\
6.75 \\
6.31 \\
6.92\end{array}$ & $\begin{array}{r}854 \\
1095 \\
874 \\
627\end{array}$ & $\begin{array}{l}5.32 \\
0.16 \\
1.49 \\
1.42\end{array}$ & $\begin{array}{l}- \\
50 \\
30 \\
45\end{array}$ & $\begin{array}{c}0 \\
0.18 \\
0.21 \\
0.14\end{array}$ & $\begin{array}{r}145 \\
37 \\
237 \\
219\end{array}$ \\
\hline MW09 & $25-35$ & $\begin{array}{l}3 / 15 / 06 \\
9 / 25 / 06 \\
3 / 27 / 07 \\
9 / 24 / 07\end{array}$ & $\begin{array}{l}17.7 \\
12.8 \\
14.9 \\
16.6\end{array}$ & $\begin{array}{l}7.33 \\
6.87 \\
6.35 \\
6.94\end{array}$ & $\begin{array}{r}664 \\
859 \\
689 \\
1999\end{array}$ & $\begin{array}{l}0.95 \\
1.59 \\
4.10 \\
3.86\end{array}$ & $\begin{array}{l}55 \\
45 \\
30 \\
55\end{array}$ & $\begin{array}{l}0.09 \\
0.18 \\
0.69 \\
0.14\end{array}$ & $\begin{array}{r}214 \\
90 \\
152 \\
186\end{array}$ \\
\hline MW10 & $30-45$ & $\begin{array}{l}3 / 14 / 06 \\
9 / 26 / 06 \\
3 / 28 / 07 \\
9 / 25 / 07\end{array}$ & $\begin{array}{l}14.8 \\
13.6 \\
17.0 \\
15.8\end{array}$ & $\begin{array}{l}6.60 \\
6.87 \\
6.36 \\
6.94\end{array}$ & $\begin{array}{r}834 \\
1058 \\
834 \\
827\end{array}$ & $\begin{array}{l}6.42 \\
6.94 \\
5.09 \\
6.64\end{array}$ & $\begin{array}{l}65 \\
50 \\
35 \\
35\end{array}$ & $\begin{array}{c}0 \\
0.50 \\
0 \\
0.21\end{array}$ & $\begin{array}{r}166 \\
51 \\
270 \\
199\end{array}$ \\
\hline SB01 & $40-50$ & $\begin{array}{r}8 / 26 / 04 \\
9 / 9 / 05 \\
10 / 12 / 05 \\
3 / 17 / 06 \\
9 / 27 / 06 \\
3 / 27 / 07 \\
9 / 27 / 07\end{array}$ & $\begin{array}{l}26.0 \\
25.0 \\
13.8 \\
12.4 \\
14.4 \\
18.0 \\
13.5\end{array}$ & $\begin{array}{l}7.46 \\
7.11 \\
7.23 \\
7.30 \\
7.03 \\
6.37 \\
7.24 \\
\end{array}$ & $\begin{array}{l}699 \\
674 \\
686 \\
692 \\
832 \\
659 \\
720\end{array}$ & $\begin{array}{c}5.21 \\
6.25 \\
- \\
5.98 \\
6.54 \\
3.81 \\
6.55\end{array}$ & $\begin{array}{c}30 \\
95 \\
- \\
55 \\
40 \\
25 \\
45\end{array}$ & $\begin{array}{c}0 \\
0 \\
- \\
0 \\
0.52 \\
0.23 \\
1.04\end{array}$ & $\begin{array}{r}210 \\
140 \\
- \\
185 \\
198 \\
173 \\
143\end{array}$ \\
\hline SB04 & $51-61$ & $\begin{array}{r}8 / 26 / 04 \\
9 / 9 / 05 \\
10 / 12 / 05 \\
3 / 16 / 06 \\
9 / 25 / 06 \\
3 / 28 / 07 \\
9 / 26 / 07\end{array}$ & $\begin{array}{l}17.9 \\
16.0 \\
13.9 \\
13.0 \\
14.9 \\
16.2 \\
19.8\end{array}$ & $\begin{array}{l}7.14 \\
7.09 \\
7.17 \\
7.57 \\
7.16 \\
6.45 \\
7.03\end{array}$ & $\begin{array}{l}765 \\
708 \\
813 \\
799 \\
791 \\
850 \\
760\end{array}$ & $\begin{array}{c}3.78 \\
8.67 \\
- \\
5.96 \\
9.32 \\
6.18 \\
6.61\end{array}$ & $\begin{array}{c}55 \\
100 \\
- \\
30 \\
70 \\
- \\
30\end{array}$ & $\begin{array}{c}0.37 \\
- \\
- \\
- \\
1.18 \\
0.23 \\
0\end{array}$ & $\begin{array}{r}230 \\
206 \\
- \\
276 \\
64 \\
266 \\
202\end{array}$ \\
\hline SB05 & $32-42$ & $\begin{array}{r}8 / 26 / 04 \\
9 / 9 / 05 \\
10 / 12 / 05 \\
3 / 17 / 06 \\
9 / 27 / 06 \\
3 / 28 / 07 \\
9 / 26 / 07\end{array}$ & $\begin{array}{l}15.7 \\
16.9 \\
14.0 \\
13.3 \\
13.7 \\
16.7 \\
15.1\end{array}$ & $\begin{array}{l}7.25 \\
6.98 \\
7.00 \\
7.67 \\
6.58 \\
4.03 \\
6.98\end{array}$ & $\begin{array}{c}761 \\
687 \\
728 \\
718 \\
763 \\
0.11 \\
810\end{array}$ & $\begin{array}{c}- \\
7.58 \\
- \\
4.80 \\
4.70 \\
2.58 \\
4.10\end{array}$ & $\begin{array}{c}25 \\
100 \\
- \\
40 \\
50 \\
35 \\
30\end{array}$ & $\begin{array}{c}0.06 \\
- \\
- \\
0.18 \\
0.25 \\
0.07 \\
0.5\end{array}$ & $\begin{array}{r}220 \\
- \\
- \\
253 \\
78 \\
296 \\
221\end{array}$ \\
\hline SB07R & $45-60$ & $\begin{array}{l}3 / 15 / 06 \\
9 / 26 / 06 \\
3 / 26 / 07 \\
9 / 25 / 07\end{array}$ & $\begin{array}{l}16.8 \\
13.2 \\
19.0 \\
17.4\end{array}$ & $\begin{array}{l}7.24 \\
6.89 \\
6.38 \\
7.06\end{array}$ & $\begin{array}{l}685 \\
842 \\
668 \\
642\end{array}$ & $\begin{array}{l}7.41 \\
6.17 \\
5.08 \\
6.30\end{array}$ & $\begin{array}{l}60 \\
55 \\
40 \\
35\end{array}$ & $\begin{array}{l}0.08 \\
0.26 \\
0.07 \\
0.11\end{array}$ & $\begin{array}{r}83 \\
67 \\
237 \\
170\end{array}$ \\
\hline SB08 & $52-62$ & $\begin{array}{c}8 / 26 / 04 \\
9 / 8 / 05 \\
10 / 12 / 05 \\
3 / 17 / 06 \\
9 / 21 / 06 \\
3 / 28 / 07 \\
9 / 26 / 07\end{array}$ & $\begin{array}{l}19.5 \\
21.2 \\
13.9 \\
12.9 \\
14.1 \\
15.8 \\
17.4\end{array}$ & $\begin{array}{l}7.31 \\
7.27 \\
7.15 \\
7.14 \\
6.96 \\
6.53 \\
7.11\end{array}$ & $\begin{array}{l}635 \\
598 \\
630 \\
645 \\
809 \\
645 \\
617\end{array}$ & $\begin{array}{c}0.16 \\
3.21 \\
- \\
3.40 \\
4.53 \\
3.57 \\
4.56\end{array}$ & $\begin{array}{c}20 \\
75 \\
- \\
40 \\
40 \\
35 \\
40\end{array}$ & $\begin{array}{c}0.53 \\
0 \\
- \\
0 \\
0 \\
0.24 \\
0.77\end{array}$ & $\begin{array}{r}235 \\
111 \\
- \\
246 \\
37 \\
208 \\
156\end{array}$ \\
\hline
\end{tabular}


TABLE 3.3 (Cont.)

\begin{tabular}{|c|c|c|c|c|c|c|c|c|c|}
\hline \multirow[b]{2}{*}{ Location } & \multirow{2}{*}{$\begin{array}{l}\text { Screen } \\
\text { Interval } \\
\text { (ft BGL) }\end{array}$} & \multirow[b]{2}{*}{$\begin{array}{c}\text { Sample } \\
\text { Date }\end{array}$} & \multirow[b]{2}{*}{$\begin{array}{c}\text { Temperature } \\
\left({ }^{\circ} \mathrm{C}\right)\end{array}$} & \multirow[b]{2}{*}{$\mathrm{pH}$} & \multirow[b]{2}{*}{$\begin{array}{l}\text { Conductivity } \\
(\mu \mathrm{S} / \mathrm{cm})\end{array}$} & \multicolumn{3}{|c|}{ Concentration (mg/L) } & \multirow[b]{2}{*}{$\begin{array}{l}\text { ORP } \\
(\mathrm{mV})\end{array}$} \\
\hline & & & & & & $\begin{array}{l}\text { Dissolved } \\
\text { Oxygen }\end{array}$ & $\begin{array}{l}\text { Carbon } \\
\text { Dioxide }\end{array}$ & Iron(II) & \\
\hline \multirow[t]{7}{*}{ SB09 } & $32-42$ & $8 / 26 / 04$ & 30.9 & 7.09 & 910 & 0.26 & 75 & 0 & 185 \\
\hline & & $9 / 11 / 05$ & 14.6 & 6.71 & 877 & 0.13 & 225 & 0 & - \\
\hline & & $10 / 11 / 05$ & 13.9 & 6.85 & 910 & - & - & - & - \\
\hline & & $3 / 17 / 06$ & 11.7 & 7.03 & 969 & 1.53 & 99 & 0 & 206 \\
\hline & & $9 / 25 / 06$ & 14.2 & 7.00 & 976 & 0.29 & 70 & 0.38 & 86 \\
\hline & & $3 / 28 / 07$ & 14.3 & 6.32 & 957 & 0.89 & 40 & 0.09 & 236 \\
\hline & & $9 / 26 / 07$ & 15.2 & 6.77 & 969 & 1.53 & 45 & 0.12 & 199 \\
\hline
\end{tabular}

${ }^{a}$ Measurement not recorded. 
TABLE 3.4 Results of attenutation parameter analyses for groundwater samples collected at Centralia, August 2004 to September 2007.

\begin{tabular}{|c|c|c|c|c|c|c|c|c|c|c|c|c|c|c|c|}
\hline \multirow[b]{2}{*}{ Well } & \multirow{2}{*}{$\begin{array}{l}\text { Screen } \\
\text { Interval } \\
\text { (ft BGL) }\end{array}$} & \multirow[b]{2}{*}{$\begin{array}{l}\text { Sample } \\
\text { Date }\end{array}$} & \multicolumn{13}{|c|}{ Concentration $(\mathrm{mg} / \mathrm{L})$} \\
\hline & & & $\begin{array}{c}\text { Total } \\
\text { Alkalinity }\end{array}$ & Chloride & Sulfate & Sulfide & Nitrate & Nitrite & $\begin{array}{l}\text { Nitrate-Nitrite } \\
\text { Nitrogen }\end{array}$ & Phosphate & Aluminum & Calcium & Iron & Magnesium & Manganese \\
\hline \multirow[t]{6}{*}{ MW01 } & $54.5-64.5$ & $8 / 24 / 04$ & 324 & 12.3 & 5.83 & - & 0.459 & $U^{a}<0.005$ & 0.299 & $U<0.2$ & $U<0.2$ & 66.2 & $U<0.1$ & 27.2 & $U<0.015$ \\
\hline & & 9/10/05 & 303 & $U<0.2$ & $U<0.2$ & 0.054 & 0.434 & $U<0.005$ & 0.299 & 0.328 & $U<0.2$ & 71.7 & $U<0.1$ & 30.2 & 0.0153 \\
\hline & & $3 / 16 / 06$ & 325 & 14.9 & 6.30 & $U<0.02$ & 0.820 & $U<0.005$ & 0.315 & $U<0.2$ & $U<0.2$ & 72.2 & $U<0.1$ & 30.1 & 0.217 \\
\hline & & $9 / 25 / 06$ & 325 & 11.6 & 5.08 & $U<0.02$ & 0.561 & - & 0.294 & $U<0.2$ & $U<0.2$ & 67.2 & $U<0.1$ & 27.6 & $U<0.015$ \\
\hline & & $3 / 29 / 07$ & 310 & 11.0 & 5.00 & $U<0.02$ & $0.330 \mathrm{H}^{\mathrm{b}}$ & - & 0.230 & $U<0.2$ & $U<0.2$ & 66.6 & $0.140 \mathrm{~B}^{\mathrm{c}}$ & 28.5 & $U<0.015$ \\
\hline & & $9 / 26 / 07$ & 310 & 11.0 & 5.10 & $U<0.02$ & 0.270 & - & 0.250 & $U<0.2$ & $U<0.2$ & 68.6 & $U<0.1$ & 30.4 & $U<0.015$ \\
\hline \multirow[t]{6}{*}{ MW02 } & $49.5-59.5$ & $8 / 26 / 04$ & 354 & 7.95 & 5.45 & - & 7.92 & 0.018 & 8.30 & $U<0.2$ & $U<0.2$ & 71.7 & $U<0.1$ & 27.5 & $U<0.015$ \\
\hline & & $9 / 11 / 05$ & 352 & 7.03 & 14.7 & 0.0563 & 9.31 & 0.028 & 9.79 & $U<0.2$ & $U<0.2$ & 71.8 & $U<0.1$ & 27.2 & 0.0167 \\
\hline & & $3 / 16 / 06$ & 364 & 8.45 & 12.2 & 0.0381 & 9.92 & 0.007 & 9.27 & 0.250 & $U<0.2$ & 73.6 & $U<0.1$ & 28.0 & $U<0.015$ \\
\hline & & 9/26/06 & 362 & 8.01 & 13.9 & $U<0.02$ & 9.73 & - & 9.25 & $U<0.2$ & $U<0.2$ & 69.0 & $U<0.1$ & 25.0 & $U<0.015$ \\
\hline & & $3 / 26 / 07$ & 360 & 8.20 & 15.0 & $U<0.02$ & $8.80 \mathrm{H}$ & - & 9.00 & $U<0.2$ & $U<0.2$ & 70.6 & $U<0.1$ & 26.0 & $U<0.015$ \\
\hline & & $9 / 26 / 07$ & 340 & 7.50 & 17.0 & $U<0.02$ & 8.00 & - & 9.10 & $U<0.2$ & $U<0.2$ & 69.4 & $U<0.1$ & 26.6 & $U<0.015$ \\
\hline \multirow[t]{6}{*}{ MW03 } & $50.5-60.5$ & $8 / 24 / 04$ & 353 & 20.6 & 8.63 & - & 6.43 & 0.0053 & 6.79 & $U<0.2$ & $U<0.2$ & 77.2 & $U<0.1$ & 27.6 & $U<0.015$ \\
\hline & & 9/10/05 & 338 & 19.6 & 7.82 & $U<0.02$ & 7.06 & 0.019 & 8.36 & 0.351 & $U<0.2$ & 78.9 & $U<0.1$ & 28.8 & $U<0.015$ \\
\hline & & $3 / 17 / 06$ & 353 & 24.0 & 9.15 & $U<0.02$ & 9.17 & $U<0.005$ & 8.59 & $U<0.2$ & $U<0.2$ & 83.2 & $U<0.1$ & 29.7 & $U<0.015$ \\
\hline & & $9 / 26 / 06$ & 356 & 25.5 & 7.82 & $U<0.02$ & 8.41 & - & 8.80 & $U<0.2$ & $U<0.2$ & 79.8 & $U<0.1$ & 28.1 & $U<0.015$ \\
\hline & & $3 / 27 / 07$ & 370 & 22.0 & 7.60 & $U<0.02$ & $7.30 \mathrm{H}$ & - & 9.70 & $U<0.2$ & $U<0.2$ & 79.1 & $U<0.1$ & 28.6 & $U<0.015$ \\
\hline & & $9 / 25 / 07$ & 330 & 25.0 & 7.60 & $U<0.02$ & $7.90 \mathrm{H}$ & - & - & $U<0.2$ & $U<0.2$ & 77.9 & $U<0.1$ & 30.0 & $U<0.015$ \\
\hline \multirow[t]{6}{*}{ MW04 } & $37.5-47.5$ & $8 / 24 / 04$ & 337 & 10.1 & 10.7 & - & 4.28 & 0.0254 & 4.42 & 0.276 & $U<0.2$ & 61.1 & $U<0.1$ & 23.3 & $U<0.015$ \\
\hline & & $9 / 11 / 05$ & 336 & 10.2 & 7.90 & $U<0.02$ & 4.45 & 0.015 & 5.05 & 0.435 & $U<0.2$ & 74.5 & $U<0.1$ & 29.4 & 0.0373 \\
\hline & & $3 / 15 / 06$ & 337 & 11.9 & 6.38 & 0.0794 & 4.97 & $U<0.005$ & 4.84 & $U<0.2$ & $U<0.2$ & 69.8 & $U<0.1$ & 27.2 & 0.0626 \\
\hline & & $9 / 25 / 06$ & 338 & 12.5 & 5.89 & $U<0.02$ & 4.51 & - & 4.31 & $U<0.2$ & $U<0.2$ & 65.0 & $U<0.1$ & 24.8 & 0.0485 \\
\hline & & $3 / 28 / 07$ & 360 & 11.0 & 5.10 & 0.027 & $3.40 \mathrm{H}$ & - & 2.60 & $U<0.2$ & $U<0.2$ & 65.7 & $U<0.1$ & 26.0 & 0.0296 \\
\hline & & $9 / 24 / 07$ & 320 & 13.0 & 5.90 & $U<0.02$ & $4.00 \mathrm{H}$ & - & 4.10 & $U<0.2$ & $U<0.2$ & 64.6 & $U<0.1$ & 26.7 & 0.0316 \\
\hline \multirow[t]{6}{*}{ MW05 } & $34.5-44.5$ & $8 / 25 / 04$ & 315 & 4.58 & 4.56 & - & 2.46 & 0.0158 & 2.55 & $U<0.2$ & $U<0.2$ & 77.2 & $U<0.1$ & 27.2 & $U<0.015$ \\
\hline & & $9 / 10 / 05$ & 303 & 9.42 & 5.35 & $U<0.02$ & 3.07 & 0.022 & 3.22 & 0.312 & $U<0.2$ & 85.4 & $U<0.1$ & 30.2 & $U<0.015$ \\
\hline & & $3 / 15 / 06$ & 304 & 9.66 & 5.17 & $U<0.02$ & 3.36 & 0.0073 & 3.20 & $U<0.2$ & $U<0.2$ & 79.8 & $U<0.1$ & 27.7 & $U<0.015$ \\
\hline & & $9 / 25 / 06$ & 319 & 7.11 & 3.90 & $U<0.02$ & 2.98 & - & 2.77 & $U<0.2$ & $U<0.2$ & 74.0 & $U<0.1$ & 25.4 & $U<0.015$ \\
\hline & & $3 / 28 / 07$ & 320 & 5.20 & 3.00 & $U<0.02$ & $2.60 \mathrm{H}$ & - & 2.00 & $U<0.2$ & $U<0.2$ & 76.8 & $U<0.1$ & 27.6 & $U<0.015$ \\
\hline & & $9 / 24 / 07$ & 300 & 8.00 & 3.80 & $U<0.02$ & $2.80 \mathrm{H}$ & - & 2.60 & $U<0.2$ & $U<0.2$ & 74.8 & $U<0.1$ & 28.2 & $U<0.015$ \\
\hline \multirow[t]{6}{*}{ MW06 } & $46.5-56.5$ & $8 / 25 / 04$ & 334 & 9.25 & 5.72 & - & 0.379 & $U<0.005$ & 0.267 & $U<0.2$ & $U<0.2$ & 73.4 & $U<0.1$ & 23.9 & $U<0.015$ \\
\hline & & $9 / 10 / 05$ & 317 & 6.88 & 4.61 & 0.0228 & 0.580 & 0.0094 & 0.345 & 0.283 & $U<0.2$ & 77.2 & $U<0.1$ & 29.8 & 0.305 \\
\hline & & $3 / 16 / 06$ & 343 & 8.98 & 5.00 & $U<0.02$ & 0.524 & 0.0055 & 0.218 & $U<0.2$ & $U<0.2$ & 73.2 & $U<0.1$ & 28.9 & 0.124 \\
\hline & & $9 / 27 / 06$ & 345 & 7.83 & 4.69 & $U<0.02$ & 0.472 & - & 0.117 & 0.681 & $U<0.2$ & 69.7 & $U<0.1$ & 26.7 & 1.30 \\
\hline & & $3 / 27 / 07$ & 330 & 8.40 & 5.20 & $U<0.02$ & $0.150 \mathrm{H}$ & - & 0.044 & $U<0.2$ & $U<0.2$ & 66.2 & $U<0.1$ & 25.1 & 0.156 \\
\hline & & $9 / 24 / 07$ & 310 & 7.30 & 4.40 & $U<0.02$ & $0.16 \mathrm{H}$ & - & $U<0.1$ & $U<0.2$ & $U<0.2$ & 63.8 & $U<0.1$ & 25.5 & 0.510 \\
\hline
\end{tabular}


TABLE 3.4 (Cont.)

\begin{tabular}{|c|c|c|c|c|c|c|c|c|c|c|c|c|c|c|c|}
\hline \multirow[b]{2}{*}{ Well } & \multirow{2}{*}{$\begin{array}{l}\text { Screen } \\
\text { Interval } \\
\text { (ft BGL) }\end{array}$} & \multirow{2}{*}{$\begin{array}{l}\text { Sample } \\
\text { Date }\end{array}$} & \multicolumn{13}{|c|}{ Concentration (mg/L) } \\
\hline & & & Alkalinity & Chloride & Sulfate & Sulfide & Nitrate & Nitrite & Nitrogen & Phosphate & Aluminum & Calcium & Iron & Magnesium & Manganese \\
\hline \multirow[t]{4}{*}{ MW07 } & $45-55$ & $3 / 14 / 06$ & 299 & 8.72 & 28.5 & $U<0.02$ & 1.18 & 0.0175 & 0.978 & $U<0.2$ & $U<0.2$ & 59.6 & $U<0.1$ & 23.1 & 0.077 \\
\hline & & $9 / 26 / 06$ & 351 & 6.03 & 23.3 & $U<0.02$ & 1.49 & - & 1.12 & 0.653 & $U<0.2$ & 66.1 & $U<0.1$ & 25.7 & $U<0.015$ \\
\hline & & $3 / 26 / 07$ & 350 & 5.90 & 20.0 & $U<0.02$ & $1.10 \mathrm{H}$ & - & 0.710 & $U<0.2$ & $U<0.2$ & 64.9 & $U<0.1$ & 26.3 & 0.0152 \\
\hline & & $9 / 24 / 07$ & 330 & 6.30 & 23.0 & $U<0.02$ & $1.10 \mathrm{H}$ & - & 0.900 & $U<0.2$ & $U<0.2$ & 63.8 & $U<0.1$ & 27.7 & $U<0.015$ \\
\hline \multirow[t]{4}{*}{ MW08 } & $38-53$ & $3 / 14 / 06$ & 342 & 47.4 & 14.4 & $U<0.02$ & 2.47 & 0.0124 & 2.14 & $U<0.2$ & $U<0.2$ & 86.5 & $U<0.1$ & 33.1 & 0.194 \\
\hline & & $9 / 26 / 06$ & 249 & 21.9 & 9.64 & $U<0.02$ & 1.44 & - & 3.10 & $U<0.2$ & $U<0.2$ & 83.1 & $U<0.1$ & 30.6 & 0.0306 \\
\hline & & $3 / 27 / 07$ & 390 & 34.0 & 11.0 & $U<0.02$ & $3.00 \mathrm{H}$ & - & 3.20 & $U<0.2$ & $U<0.2$ & 85.0 & $U<0.1$ & 32.1 & $U<0.015$ \\
\hline & & $9 / 25 / 07$ & 390 & 40.0 & 10.0 & $U<0.02$ & $2.80 \mathrm{H}$ & - & - & $U<0.2$ & $U<0.2$ & 82.3 & $U<0.1$ & 33.0 & $U<0.015$ \\
\hline \multirow[t]{4}{*}{ MW09 } & $25-35$ & $3 / 15 / 06$ & 329 & 6.39 & 6.23 & $U<0.02$ & 3.25 & 0.0203 & 2.93 & $U<0.2$ & $U<0.2$ & 83.2 & $U<0.1$ & 28.8 & 0.133 \\
\hline & & $9 / 25 / 06$ & 360 & 7.04 & 5.41 & $U<0.02$ & 3.86 & - & 3.54 & $U<0.2$ & $U<0.2$ & 85.5 & $U<0.1$ & 29.5 & $U<0.015$ \\
\hline & & $3 / 27 / 07$ & 360 & 6.90 & 6.40 & $U<0.02$ & $4.10 \mathrm{H}$ & - & 3.80 & $U<0.2$ & $U<0.2$ & 87.4 & $U<0.1$ & 31.3 & $U<0.015$ \\
\hline & & $9 / 24 / 07$ & 340 & 7.30 & 7.50 & $U<0.02$ & $3.80 \mathrm{H}$ & - & 3.70 & $U<0.2$ & $U<0.2$ & 87.5 & $U<0.1$ & 33.2 & $U<0.015$ \\
\hline \multirow[t]{4}{*}{ MW10 } & $30-45$ & $3 / 14 / 06$ & 298 & 74.3 & 10.8 & $U<0.02$ & 1.23 & 0.0175 & 0.814 & $U<0.2$ & $U<0.2$ & 91.6 & $U<0.1$ & 32.0 & 0.129 \\
\hline & & $9 / 26 / 06$ & 319 & 97.9 & 8.80 & $U<0.02$ & 1.22 & - & 1.34 & 0.606 & $U<0.2$ & 88.4 & $U<0.1$ & 29.7 & $U<0.015$ \\
\hline & & $3 / 28 / 07$ & 330 & 100 & 9.10 & $U<0.02$ & $1.20 \mathrm{H}$ & - & 1.10 & $U<0.2$ & $U<0.2$ & 89.4 & $U<0.1$ & 31.1 & $U<0.015$ \\
\hline & & $9 / 25 / 07$ & 310 & 97.0 & 8.90 & $U<0.02$ & $0.99 \mathrm{H}$ & - & - & $U<0.2$ & $U<0.2$ & 88.8 & $U<0.1$ & 32.4 & $U<0.015$ \\
\hline \multirow[t]{6}{*}{ SB01 } & $40-50$ & $8 / 26 / 04$ & 292 & 61.8 & 10.6 & - & 2.32 & $U<0.005$ & 1.94 & $U<0.2$ & $U<0.2$ & 75.0 & $U<0.1$ & 30.6 & 0.106 \\
\hline & & 9/9/05 & 339 & 18.9 & 4.31 & $U<0.02$ & 1.05 & 0.0082 & 1.86 & $U<0.2$ & $U<0.2$ & 75.2 & $U<0.1$ & 30.3 & $U<0.015$ \\
\hline & & $3 / 17 / 06$ & 338 & 22.5 & 4.87 & $U<0.02$ & 1.14 & - & 2.50 & $U<0.2$ & $U<0.2$ & 66.1 & $U<0.1$ & 26.5 & $U<0.015$ \\
\hline & & $9 / 27 / 06$ & 336 & 60.6 & 46.2 & $\mathrm{U}<0.02$ & 3.34 & - & 3.46 & 0.233 & $U<0.2$ & 74.6 & $U<0.1$ & 28.5 & $U<0.015$ \\
\hline & & $3 / 27 / 07$ & 340 & 30.0 & 7.40 & $U<0.02$ & $1.10 \mathrm{H}$ & - & 0.800 & $U<0.2$ & $U<0.2$ & 67.9 & $U<0.1$ & 27.3 & $U<0.015$ \\
\hline & & 9/27/07 & 320 & 34.0 & 8.30 & $U<0.02$ & 1.30 & - & - & $U<0.2$ & $U<0.2$ & 72.6 & $U<0.1$ & 30.4 & $U<0.015$ \\
\hline \multirow[t]{6}{*}{ SB04 } & $51-61$ & $8 / 26 / 04$ & 375 & 40.3 & 5.89 & - & 1.84 & $U<0.005$ & 1.93 & $U<0.2$ & $U<0.2$ & 78.8 & $U<0.1$ & 30.2 & $U<0.015$ \\
\hline & & 9/9/05 & 369 & 45.5 & 5.05 & $U<0.02$ & 1.73 & 0.0077 & 2.64 & $U<0.2$ & $U<0.2$ & 79.0 & $U<0.1$ & 30.0 & $U<0.015$ \\
\hline & & $3 / 16 / 06$ & 371 & 40.0 & 5.98 & $U<0.02$ & 3.07 & $U<0.005$ & 2.85 & $U<0.2$ & $U<0.2$ & 82.6 & $U<0.1$ & 31.4 & $U<0.015$ \\
\hline & & 9/25/06 & 374 & 45.1 & 6.67 & $U<0.02$ & 2.44 & - & 2.56 & $U<0.2$ & $U<0.2$ & 76.0 & $U<0.1$ & 27.7 & $U<0.015$ \\
\hline & & $3 / 28 / 07$ & 370 & 48.0 & 15.0 & $U<0.02$ & $1.70 \mathrm{H}$ & - & 4.60 & $U<0.2$ & $U<0.2$ & 79.1 & $U<0.1$ & 28.6 & $U<0.015$ \\
\hline & & $9 / 26 / 07$ & 370 & 42.0 & 8.50 & $U<0.02$ & 1.30 & - & 2.00 & $U<0.2$ & $U<0.2$ & 77.4 & $U<0.1$ & 29.5 & $U<0.015$ \\
\hline \multirow[t]{6}{*}{ SB05 } & $32-42$ & $8 / 26 / 04$ & 326 & 51.9 & 6.07 & - & 2.42 & $U<0.005$ & 2.28 & $U<0.2$ & $U<0.2$ & 98.5 & $U<0.1$ & 37.0 & $U<0.015$ \\
\hline & & 9/9/05 & 318 & 57.4 & 4.27 & $U<0.02$ & 2.57 & 0.010 & 2.41 & $U<0.2$ & $U<0.2$ & 88.1 & $U<0.1$ & 33.0 & $U<0.015$ \\
\hline & & $3 / 17 / 06$ & 324 & 57.1 & 2.96 & $U<0.02$ & 2.56 & $U<0.005$ & 2.73 & $U<0.2$ & $U<0.2$ & 92.6 & $U<0.1$ & 35.0 & $U<0.015$ \\
\hline & & 9/27/06 & 320 & 67.1 & 4.11 & $\mathrm{U}<0.02$ & 2.33 & - & 2.47 & 0.521 & $U<0.2$ & 86.6 & $U<0.1$ & 31.1 & $U<0.015$ \\
\hline & & $3 / 28 / 07$ & 320 & 66.0 & 4.90 & $U<0.02$ & $2.40 \mathrm{H}$ & - & 2.10 & $U<0.2$ & $U<0.2$ & 67.7 & $U<0.1$ & 23.4 & $U<0.015$ \\
\hline & & $9 / 26 / 07$ & 300 & 41.0 & 14.0 & $U<0.02$ & 2.00 & - & 2.30 & $U<0.2$ & $U<0.2$ & 93.4 & $U<0.1$ & 37.2 & $U<0.015$ \\
\hline
\end{tabular}


TABLE 3.4 (Cont.)

\begin{tabular}{|c|c|c|c|c|c|c|c|c|c|c|c|c|c|c|c|}
\hline \multirow[b]{2}{*}{ Well } & \multirow[b]{2}{*}{$\begin{array}{l}\text { Screen } \\
\text { Interval } \\
\text { (ft BGL) }\end{array}$} & \multirow[b]{2}{*}{$\begin{array}{l}\text { Sample } \\
\text { Date }\end{array}$} & \multicolumn{13}{|c|}{ Concentration $(\mathrm{mg} / \mathrm{L})$} \\
\hline & & & $\begin{array}{c}\text { Total } \\
\text { Alkalinity }\end{array}$ & Chloride & Sulfate & Sulfide & Nitrate & Nitrite & $\begin{array}{l}\text { Nitrate-Nitrite } \\
\text { Nitrogen }\end{array}$ & Phosphate & Aluminum & Calcium & Iron & Magnesium & Manganese \\
\hline \multirow[t]{4}{*}{ SB07R } & $45-60$ & $3 / 15 / 06$ & 318 & 30.4 & 16.8 & $U<0.02$ & 1.27 & 0.0084 & 1.04 & $U<0.2$ & $U<0.2$ & 72.4 & $U<0.1$ & 26.0 & 0.179 \\
\hline & & $9 / 26 / 06$ & 333 & 25.2 & 17.2 & $U<0.02$ & 1.47 & - & 1.62 & 0.612 & $U<0.2$ & 71.0 & $U<0.1$ & 25.1 & $U<0.015$ \\
\hline & & $3 / 26 / 07$ & 310 & 23.0 & 11.0 & $U<0.02$ & $1.00 \mathrm{H}$ & - & 1.10 & $U<0.2$ & $U<0.2$ & 70.2 & $U<0.1$ & 25.4 & $U<0.015$ \\
\hline & & $9 / 25 / 07$ & 300 & 27.0 & 14.0 & $U<0.02$ & $1.10 \mathrm{H}$ & - & - & $U<0.2$ & $U<0.2$ & 69.8 & $U<0.1$ & 27.1 & $U<0.015$ \\
\hline \multirow[t]{6}{*}{ SB08 } & $52-62$ & $8 / 26 / 04$ & 322 & 17.9 & 8.51 & - & 1.12 & $U<0.005$ & 0.892 & $U<0.2$ & $U<0.2$ & 74.9 & $U<0.1$ & 30.1 & $U<0.015$ \\
\hline & & $9 / 8 / 05$ & 319 & 16.2 & 7.83 & $U<0.02$ & 1.37 & 0.012 & 1.28 & $U<0.2$ & $U<0.2$ & 73.0 & $U<0.1$ & 29.2 & $U<0.015$ \\
\hline & & $3 / 17 / 06$ & 327 & 19.3 & 9.25 & $U<0.02$ & 1.69 & $U<0.005$ & 1.41 & $U<0.2$ & $U<0.2$ & 72.7 & $U<0.1$ & 29.1 & $U<0.015$ \\
\hline & & $9 / 21 / 06$ & 323 & 16.9 & 7.08 & $U<0.02$ & 1.34 & - & 1.15 & $U<0.2$ & $U<0.2$ & 69.5 & $U<0.1$ & 27.4 & $U<0.015$ \\
\hline & & $3 / 28 / 07$ & 330 & 17.0 & 7.40 & $U<0.02$ & $1.20 \mathrm{H}$ & - & 0.68 & $U<0.2$ & $U<0.2$ & 71.9 & $U<0.1$ & 29.0 & $U<0.015$ \\
\hline & & $9 / 26 / 07$ & 300 & 14.0 & 6.90 & $U<0.02$ & 0.940 & - & 1.10 & $U<0.2$ & $U<0.2$ & 71.7 & $U<0.1$ & 30.1 & $U<0.015$ \\
\hline \multirow[t]{6}{*}{ SB09 } & $32-42$ & $8 / 26 / 04$ & 449 & 14.8 & 32.0 & - & 4.92 & 0.009 & 5.08 & $U<0.2$ & $U<0.2$ & 108 & $U<0.1$ & 39.1 & $U<0.015$ \\
\hline & & $9 / 11 / 05$ & 446 & 13.4 & 30.1 & $U<0.02$ & 4.37 & 0.021 & 4.91 & $U<0.2$ & $U<0.2$ & 119 & $U<0.1$ & 42.2 & $U<0.015$ \\
\hline & & $3 / 17 / 06$ & 495 & 15.6 & 38.8 & $U<0.02$ & 4.67 & $U<0.005$ & 4.76 & $U<0.2$ & $U<0.2$ & 115 & $U<0.1$ & 40.4 & $U<0.015$ \\
\hline & & $9 / 25 / 06$ & 504 & 15.5 & 34.5 & $U<0.02$ & 4.17 & - & 4.13 & $U<0.2$ & $U<0.2$ & 108 & $U<0.1$ & 36.5 & $U<0.015$ \\
\hline & & $3 / 28 / 07$ & 530 & 16.0 & 32.0 & $U<0.02$ & $4.40 \mathrm{H}$ & - & 1.20 & $U<0.2$ & $U<0.2$ & 112 & $U<0.1$ & 39.5 & $U<0.015$ \\
\hline & & $9 / 26 / 07$ & 480 & 16.0 & 30.0 & $U<0.02$ & 3.90 & - & 4.00 & $U<0.2$ & $U<0.2$ & 110 & $U<0.1$ & 40.3 & $U<0.015$ \\
\hline
\end{tabular}

\begin{tabular}{|c|c|c|c|c|c|c|c|c|c|c|c|c|c|}
\hline \multirow[b]{2}{*}{ Well } & \multirow{2}{*}{$\begin{array}{l}\text { Screen } \\
\text { Interval } \\
\text { (ft BGL) }\end{array}$} & \multirow{2}{*}{$\begin{array}{l}\text { Sample } \\
\text { Date }\end{array}$} & \multicolumn{5}{|c|}{ Concentration (mg/L) } & \multicolumn{3}{|c|}{ Concentration $(\mu \mathrm{g} / \mathrm{L})$} & \multirow{2}{*}{$\begin{array}{c}\text { Total } \\
\text { Organic } \\
\text { Carbon } \\
(\mathrm{mg} / \mathrm{L})\end{array}$} & \multirow{2}{*}{$\begin{array}{c}\text { Dissolved } \\
\text { Hydrogen } \\
\text { (nM) }\end{array}$} & \multirow{2}{*}{$\begin{array}{c}\text { Tritium } \\
\text { (TU) }\end{array}$} \\
\hline & & & Phosphorus & Potassium & Silicon & Sodium & Zinc & Ethane & Ethene & Methane & & & \\
\hline \multirow[t]{6}{*}{ MW01 } & $54.5-64.5$ & $8 / 24 / 04$ & - & $U<5$ & 14.9 & 23.4 & $U<0.02$ & $<4$ & $<3$ & $<2$ & $<1$ & - & $0.11 \pm 0.09$ \\
\hline & & 9/10/05 & $U<0.25$ & $U<5$ & $31.6 \mathrm{E}^{\mathrm{d}}$ & 24.6 & $U<0.02$ & $<4$ & $<3$ & $<2$ & $<1$ & - & \\
\hline & & $3 / 16 / 06$ & $U<0.25$ & $U<5$ & 15.2 & 24.8 & $U<0.02$ & $<4$ & $<3$ & $<2$ & 6.19 & - & \\
\hline & & $9 / 25 / 06$ & $U<0.25$ & $U<5$ & 15.8 & 22.8 & $U<0.02$ & $<4$ & $<3$ & $<2$ & $<1$ & - & \\
\hline & & $3 / 29 / 07$ & $U<0.25$ & $U<5$ & 16.1 & 24.0 & $U<0.02$ & $<4$ & $<3$ & $<2$ & $<1$ & - & \\
\hline & & 9/26/07 & $U<0.25$ & $U<5$ & 16.7 & 24.8 & $U<0.02$ & $<4$ & $<3$ & $<2$ & 1.50 & - & \\
\hline \multirow[t]{6}{*}{ MW02 } & $49.5-59.5$ & $8 / 26 / 04$ & $U<0.25$ & $U<5$ & 15.1 & 49.8 & $U<0.02$ & $<4$ & $<3$ & $<2$ & 1.84 & - & $0.78 \pm 0.09$ \\
\hline & & $9 / 11 / 05$ & $U<0.25$ & $U<5$ & 15.9 & 56.4 & $U<0.02$ & $<4$ & $<3$ & 59 & $<1$ & 3.1 & \\
\hline & & $3 / 16 / 06$ & $U<0.25$ & $U<5$ & 16.4 & 56.1 & $U<0.02$ & $<4$ & $<3$ & 34 & 3.57 & - & \\
\hline & & 9/26/06 & $U<0.25$ & $U<5$ & 15.4 & 53.0 & $U<0.02$ & $<4$ & $<3$ & 43 & 1.29 & - & \\
\hline & & $3 / 26 / 07$ & $U<0.25$ & $U<5$ & 15.8 & 55.1 & $U<0.02$ & $<4$ & $<3$ & 21 & 1.10 & - & \\
\hline & & 9/26/07 & $U<0.25$ & $U<5$ & 14.4 & 57.0 & $U<0.02$ & $<4$ & $<3$ & 13 & 1.30 & - & \\
\hline
\end{tabular}


TABLE 3.4 (Cont.)

\begin{tabular}{|c|c|c|c|c|c|c|c|c|c|c|c|c|c|}
\hline \multirow[b]{2}{*}{ Well } & \multirow{2}{*}{$\begin{array}{l}\text { Screen } \\
\text { Interval } \\
\text { (ft BGL) }\end{array}$} & \multirow{2}{*}{$\begin{array}{l}\text { Sample } \\
\text { Date }\end{array}$} & \multicolumn{5}{|c|}{ Concentration $(\mathrm{mg} / \mathrm{L})$} & \multicolumn{3}{|c|}{ Concentration $(\mu \mathrm{g} / \mathrm{L})$} & \multirow{2}{*}{$\begin{array}{c}\text { Total } \\
\text { Organic } \\
\text { Carbon } \\
(\mathrm{mg} / \mathrm{L})\end{array}$} & \multirow{2}{*}{$\begin{array}{c}\text { Dissolved } \\
\text { Hydrogen } \\
\text { (nM) }\end{array}$} & \multirow{2}{*}{$\begin{array}{c}\text { Tritium } \\
\text { (TU) }\end{array}$} \\
\hline & & & Phosphorus & Potassium & Silicon & Sodium & Zinc & Ethane & Ethene & Methane & & & \\
\hline \multirow[t]{6}{*}{ MW03 } & $50.5-60.5$ & $8 / 24 / 04$ & - & $U<5$ & 15.1 & 44.7 & $U<0.02$ & $<4$ & $<3$ & $<2$ & 1.14 & - & $0.09 \pm 0.09$ \\
\hline & & $9 / 10 / 05$ & $U<0.25$ & $U<5$ & $30.9 \mathrm{E}$ & 44.4 & $U<0.02$ & $<4$ & $<3$ & $<2$ & $<1$ & - & \\
\hline & & $3 / 17 / 06$ & $U<0.25$ & $U<5$ & 15.0 & 46.8 & $U<0.02$ & $<4$ & $<3$ & $<2$ & 1.23 & - & \\
\hline & & $9 / 26 / 06$ & $U<0.25$ & $U<5$ & 15.9 & 44.0 & $U<0.02$ & $<4$ & $<3$ & $<2$ & 1.02 & - & \\
\hline & & $3 / 27 / 07$ & $U<0.25$ & $U<5$ & 15.8 & 44.5 & $U<0.02$ & $<4$ & $<3$ & $<2$ & $<1$ & - & \\
\hline & & $9 / 25 / 07$ & $U<0.25$ & $U<5$ & 15.6 & 44.2 & $U<0.02$ & $<4$ & $<3$ & $<2$ & 1.50 & - & \\
\hline \multirow[t]{6}{*}{ MW04 } & $37.5-47.5$ & $8 / 24 / 04$ & - & $U<5$ & 15.4 & 59.3 & $U<0.02$ & $<4$ & $<3$ & $<2$ & $<1$ & - & $0.15 \pm 0.09$ \\
\hline & & $9 / 11 / 05$ & $U<0.25$ & $U<5$ & 15.5 & 48.0 & $U<0.02$ & $<4$ & $<3$ & 4.5 & $<1$ & - & \\
\hline & & $3 / 15 / 06$ & $U<0.25$ & $U<5$ & 15.1 & 43.8 & $U<0.02$ & $<4$ & $<3$ & 51 & 5.07 & - & \\
\hline & & $9 / 25 / 06$ & $U<0.25$ & $U<5$ & 14.9 & 38.3 & $U<0.02$ & $<4$ & $<3$ & 140 & $<1$ & - & \\
\hline & & $3 / 28 / 07$ & $U<0.25$ & $U<5$ & 15.3 & 40.5 & $U<0.02$ & $<4$ & $<3$ & 6.2 & $<1$ & - & \\
\hline & & $9 / 24 / 07$ & $U<0.25$ & $U<5$ & 14.0 & 40.2 & 0.057 & $<4$ & $<3$ & 6.8 & $<1$ & - & \\
\hline \multirow[t]{6}{*}{ MW05 } & $34.5-44.5$ & $8 / 25 / 04$ & - & $U<5$ & 15.1 & 13.5 & $U<0.02$ & $<4$ & $<3$ & $<2$ & $<1$ & - & $0.16 \pm 0.09$ \\
\hline & & $9 / 10 / 05$ & $U<0.25$ & $U<5$ & 15.6 & 12.5 & $U<0.02$ & $<4$ & $<3$ & $<2$ & $<1$ & - & \\
\hline & & $3 / 15 / 06$ & $U<0.25$ & $U<5$ & 14.2 & 11.6 & $U<0.02$ & $<4$ & $<3$ & $<2$ & 5.54 & - & \\
\hline & & $9 / 25 / 06$ & $U<0.25$ & $U<5$ & 14.6 & 9.43 & $U<0.02$ & $<4$ & $<3$ & $<2$ & $<1$ & - & \\
\hline & & $3 / 28 / 07$ & $U<0.25$ & $U<5$ & 15.5 & 10.6 & $U<0.02$ & $<4$ & $<3$ & $<2$ & $<1$ & - & \\
\hline & & $9 / 24 / 07$ & $U<0.25$ & $U<5$ & 15.4 & 9.96 & $U<0.02$ & $<4$ & $<3$ & $<2$ & 1.20 & - & \\
\hline \multirow[t]{6}{*}{ MW06 } & $46.5-56.5$ & $8 / 25 / 04$ & - & $U<5$ & 13.6 & 26.7 & $U<0.02$ & $<4$ & $<3$ & $<2$ & 1.62 & - & $0.10 \pm 0.09$ \\
\hline & & $9 / 10 / 05$ & $U<0.25$ & $U<5$ & 15.6 & 27.1 & $U<0.02$ & $<4$ & $<3$ & $<2$ & $<1$ & - & \\
\hline & & $3 / 16 / 06$ & $U<0.25$ & $U<5$ & 15.5 & 25.8 & $U<0.02$ & $<4$ & $<3$ & 2.3 & 4.12 & - & \\
\hline & & $9 / 27 / 06$ & $U<0.25$ & $U<5$ & 14.1 & 22.8 & $U<0.02$ & $<4$ & $<3$ & 65 & $<1$ & - & \\
\hline & & $3 / 27 / 07$ & $U<0.25$ & $U<5$ & 14.2 & 23.0 & $U<0.02$ & $<4$ & $<3$ & 6.5 & 4.00 & - & \\
\hline & & $9 / 24 / 07$ & $U<0.25$ & $U<5$ & 14.0 & 21.7 & $U<0.02$ & $<4$ & $<3$ & 74 & 1.50 & - & \\
\hline \multirow[t]{4}{*}{ MW07 } & $45-55$ & $3 / 14 / 06$ & $U<0.25$ & $U<5$ & 10.5 & 33.3 & $U<0.02$ & $<4$ & $<3$ & $<2$ & 35.4 & - & $0.31 \pm 0.09$ \\
\hline & & $9 / 26 / 06$ & $U<0.25$ & $U<5$ & 12.7 & 34.9 & $U<0.02$ & $<4$ & $<3$ & $<2$ & 2.24 & - & \\
\hline & & $3 / 26 / 07$ & $U<0.25$ & $U<5$ & 13.6 & 35.6 & 0.197 & $<4$ & $<3$ & $<2$ & 1.00 & - & \\
\hline & & $9 / 24 / 07$ & $U<0.25$ & $U<5$ & 13.5 & 35.9 & 0.102 & $<4$ & $<3$ & $<2$ & $<1$ & - & \\
\hline \multirow[t]{4}{*}{ MW08 } & $38-53$ & $3 / 14 / 06$ & $U<0.25$ & $U<5$ & 15.8 & 57.5 & $U<0.02$ & $<4$ & $<3$ & $<2$ & 9.00 & - & $4.63 \pm 0.15$ \\
\hline & & $9 / 26 / 06$ & $U<0.25$ & $U<5$ & 14.4 & 51.9 & 0.0239 & $<4$ & $<3$ & $<2$ & 1.96 & - & \\
\hline & & $3 / 27 / 07$ & $U<0.25$ & $U<5$ & 14.7 & 53.8 & $U<0.02$ & $<4$ & $<3$ & $<2$ & 1.90 & - & \\
\hline & & $9 / 25 / 07$ & $U<0.25$ & $U<5$ & 14.8 & 54.2 & $U<0.02$ & $<4$ & $<3$ & $<2$ & 4.80 & - & \\
\hline \multirow[t]{4}{*}{ MW09 } & $25-35$ & $3 / 15 / 06$ & $U<0.25$ & $U<5$ & 13.6 & 11.6 & $U<0.02$ & $<4$ & $<3$ & $<2$ & 10.7 & - & $1.87 \pm 0.09$ \\
\hline & & $9 / 25 / 06$ & $U<0.25$ & $U<5$ & 14.6 & 11.5 & $U<0.02$ & $<4$ & $<3$ & $<2$ & $<1$ & - & \\
\hline & & $3 / 27 / 07$ & $U<0.25$ & $U<5$ & 15.0 & 13.2 & $U<0.02$ & $<4$ & $<3$ & $<2$ & $<1$ & - & \\
\hline & & $9 / 24 / 07$ & $U<0.25$ & $U<5$ & 15.7 & 13.2 & $U<0.02$ & $<4$ & $<3$ & $<2$ & 1.10 & - & \\
\hline
\end{tabular}


TABLE 3.4 (Cont.)

\begin{tabular}{|c|c|c|c|c|c|c|c|c|c|c|c|c|c|}
\hline \multirow[b]{2}{*}{ Well } & \multirow{2}{*}{$\begin{array}{l}\text { Screen } \\
\text { Interval } \\
\text { (ft BGL) }\end{array}$} & \multirow{2}{*}{$\begin{array}{l}\text { Sample } \\
\text { Date }\end{array}$} & \multicolumn{5}{|c|}{ Concentration (mg/L) } & \multicolumn{3}{|c|}{ Concentration $(\mu \mathrm{g} / \mathrm{L})$} & \multirow{2}{*}{$\begin{array}{c}\text { Total } \\
\text { Organic } \\
\text { Carbon } \\
(\mathrm{mg} / \mathrm{L})\end{array}$} & \multirow{2}{*}{$\begin{array}{c}\text { Dissolved } \\
\text { Hydrogen } \\
\text { (nM) }\end{array}$} & \multirow{2}{*}{$\begin{array}{c}\text { Tritium } \\
\text { (TU) }\end{array}$} \\
\hline & & & Phosphorus & Potassium & Silicon & Sodium & Zinc & Ethane & Ethene & Methane & & & \\
\hline \multirow[t]{4}{*}{ MW10 } & $30-45$ & $3 / 14 / 06$ & $U<0.25$ & $U<5$ & 14.5 & 38.7 & $U<0.02$ & $<4$ & $<3$ & $<2$ & 7.96 & - & $1.39 \pm 0.09$ \\
\hline & & 9/26/06 & $U<0.25$ & $U<5$ & 13.4 & 41.9 & $U<0.02$ & $<4$ & $<3$ & $<2$ & 1.69 & - & \\
\hline & & 3/28/07 & $U<0.25$ & $U<5$ & 14.0 & 45.0 & $U<0.02$ & $<4$ & $<3$ & $<2$ & 1.20 & - & \\
\hline & & $9 / 25 / 07$ & $U<0.25$ & $U<5$ & 15.2 & 41.9 & 0.0477 & $<4$ & $<3$ & $<2$ & $<1$ & - & \\
\hline \multirow[t]{6}{*}{ SB01 } & $40-50$ & 8/26/04 & $U<0.25$ & $U<5$ & 14.2 & 44.0 & $U<0.02$ & $<4$ & $<3$ & $<2$ & 4.04 & - & \\
\hline & & 9/9/05 & $U<0.25$ & $U<5$ & $31.7 \mathrm{E}$ & 44.1 & $U<0.02$ & $<4$ & $<3$ & $<2$ & 1.86 & 71 & \\
\hline & & $3 / 17 / 06$ & $U<0.25$ & $U<5$ & 16.1 & 35.8 & $U<0.02$ & $<4$ & $<3$ & $<2$ & 8.97 & - & \\
\hline & & 9/27/06 & $U<0.25$ & $U<5$ & 14.6 & 47.7 & 0.0339 & $<4$ & $<3$ & $<2$ & 1.59 & - & \\
\hline & & $3 / 27 / 07$ & $U<0.25$ & $U<5$ & 15.5 & 36.7 & $U<0.02$ & $<4$ & $<3$ & $<2$ & $<1$ & - & \\
\hline & & $9 / 27 / 07$ & $U<0.25$ & $U<5$ & 15.5 & 47.1 & $U<0.02$ & $<4$ & $<3$ & $<2$ & 1.70 & - & \\
\hline \multirow[t]{6}{*}{ SB04 } & $51-61$ & $8 / 26 / 04$ & $U<0.25$ & $U<5$ & 15.4 & 57.8 & $U<0.02$ & $<4$ & $<3$ & $<2$ & 3.00 & - & \\
\hline & & 9/9/05 & $U<0.25$ & $U<5$ & 15.0 & 54.9 & $U<0.02$ & $<4$ & $<3$ & $<2$ & $<1$ & 24 & \\
\hline & & 3/16/06 & $U<0.25$ & $U<5$ & 16.5 & 56.9 & $U<0.02$ & $<4$ & $<3$ & $<2$ & 3.78 & - & \\
\hline & & 9/25/06 & $U<0.25$ & $U<5$ & 15.6 & 51.2 & $U<0.02$ & $<4$ & $<3$ & $<2$ & 1.20 & - & \\
\hline & & $3 / 28 / 07$ & $U<0.25$ & $U<5$ & 16.1 & 62.2 & $U<0.02$ & $<4$ & $<3$ & $<2$ & 2.00 & - & \\
\hline & & 9/26/07 & $U<0.25$ & $U<5$ & 16.4 & 58.0 & $U<0.02$ & $<4$ & $<3$ & $<2$ & 1.20 & - & \\
\hline \multirow[t]{6}{*}{ SB05 } & $32-42$ & $8 / 26 / 04$ & $U<0.25$ & $U<5$ & 15.3 & 34.0 & $U<0.02$ & $<4$ & $<3$ & $<2$ & 2.26 & - & \\
\hline & & $9 / 9 / 05$ & $U<0.25$ & $U<5$ & $33.4 \mathrm{E}$ & 20.3 & $U<0.02$ & $<4$ & $<3$ & $<2$ & $<1$ & 11 & \\
\hline & & $3 / 17 / 06$ & $U<0.25$ & $U<5$ & 14.5 & 21.3 & $\mathrm{U}<0.02$ & $<4$ & $<3$ & $<2$ & 4.97 & - & \\
\hline & & 9/27/06 & $U<0.25$ & $U<5$ & 15.0 & 21.9 & $\mathrm{U}<0.02$ & $<4$ & $<3$ & $<2$ & 1.17 & - & \\
\hline & & 3/28/07 & $U<0.25$ & $U<5$ & 16.6 & 52.3 & $U<0.02$ & $<4$ & $<3$ & $<2$ & 1.20 & - & \\
\hline & & $9 / 26 / 07$ & $U<0.25$ & $U<5$ & 15.7 & 24.5 & $U<0.02$ & $<4$ & $<3$ & $<2$ & 1.60 & - & \\
\hline \multirow[t]{4}{*}{ SB07R } & $45-60$ & $3 / 15 / 06$ & $U<0.25$ & $U<5$ & 13.8 & 38.2 & $U<0.02$ & $<4$ & $<3$ & $<2$ & 11.2 & - & $0.32 \pm 0.09$ \\
\hline & & 9/26/06 & $U<0.25$ & $U<5$ & 14.0 & 34.5 & 0.0371 & $<4$ & $<3$ & $<2$ & 1.35 & - & \\
\hline & & $3 / 26 / 07$ & $U<0.25$ & $U<5$ & 14.1 & 32.5 & $U<0.02$ & $<4$ & $<3$ & $<2$ & 1.10 & - & \\
\hline & & $9 / 25 / 07$ & $U<0.25$ & $U<5$ & 14.9 & 33.7 & 0.0546 & $<4$ & $<3$ & $<2$ & 1.00 & - & \\
\hline \multirow[t]{6}{*}{ SB08 } & $52-62$ & $8 / 26 / 04$ & $U<0.25$ & $U<5$ & 15.9 & 27.1 & $U<0.02$ & $<4$ & $<3$ & $<2$ & 1.88 & - & \\
\hline & & $9 / 8 / 05$ & $U<0.25$ & $U<5$ & 14.9 & 26.7 & $U<0.02$ & $<4$ & $<3$ & $<2$ & $<1$ & 6.1 & \\
\hline & & $3 / 17 / 06$ & $U<0.25$ & $U<5$ & 14.6 & 26.1 & $U<0.02$ & $<4$ & $<3$ & $<2$ & 5.99 & - & \\
\hline & & $9 / 21 / 06$ & $U<0.25$ & $U<5$ & 16.1 & 24.4 & $U<0.02$ & $<4$ & $<3$ & $<2$ & $<1$ & - & \\
\hline & & $3 / 28 / 07$ & $U<0.25$ & $U<5$ & 16.2 & 26.6 & $U<0.02$ & $<4$ & $<3$ & $<2$ & $<1$ & - & \\
\hline & & 9/26/07 & $U<0.25$ & $U<5$ & 16.7 & 26.8 & $U<0.02$ & $<4$ & $<3$ & $<2$ & 1.30 & - & \\
\hline
\end{tabular}


TABLE 3.4 (Cont.)

\begin{tabular}{|c|c|c|c|c|c|c|c|c|c|c|c|c|c|}
\hline \multirow[b]{2}{*}{ Well } & \multirow{2}{*}{$\begin{array}{l}\text { Screen } \\
\text { Interval } \\
\text { (ft BGL) }\end{array}$} & \multirow{2}{*}{$\begin{array}{c}\text { Sample } \\
\text { Date }\end{array}$} & \multicolumn{5}{|c|}{ Concentration (mg/L) } & \multicolumn{3}{|c|}{ Concentration $(\mu \mathrm{g} / \mathrm{L})$} & \multirow{2}{*}{$\begin{array}{c}\text { Total } \\
\text { Organic } \\
\text { Carbon } \\
(\mathrm{mg} / \mathrm{L})\end{array}$} & \multirow{2}{*}{$\begin{array}{l}\text { Dissolved } \\
\text { Hydrogen } \\
\text { (nM) }\end{array}$} & \multirow{2}{*}{$\begin{array}{c}\text { Tritium } \\
\text { (TU) }\end{array}$} \\
\hline & & & Phosphorus & Potassium & Silicon & Sodium & Zinc & Ethane & Ethene & Methane & & & \\
\hline \multirow[t]{6}{*}{ SB09 } & $32-42$ & $8 / 26 / 04$ & $U<0.25$ & $U<5$ & 14.7 & 52.5 & $U<0.02$ & $<4$ & $<3$ & $<2$ & 1.94 & - & \\
\hline & & $9 / 11 / 05$ & $U<0.25$ & $U<5$ & 15.0 & 57.0 & $U<0.02$ & $<4$ & $<3$ & $<2$ & 1.57 & - & \\
\hline & & $3 / 17 / 06$ & $U<0.25$ & $U<5$ & 14.2 & 54.9 & $U<0.02$ & $<4$ & $<3$ & $<2$ & 6.88 & - & \\
\hline & & $9 / 25 / 06$ & $U<0.25$ & $U<5$ & 15.0 & 49.5 & $U<0.02$ & $<4$ & $<3$ & $<2$ & 1.86 & - & \\
\hline & & $3 / 28 / 07$ & $U<0.25$ & $U<5$ & 16.0 & 53.2 & $U<0.02$ & $<4$ & $<3$ & $<2$ & 1.50 & - & \\
\hline & & 9/26/07 & $U<0.25$ & $U<5$ & 15.0 & 52.1 & $U<0.02$ & $<4$ & $<3$ & $<2$ & 3.70 & - & \\
\hline
\end{tabular}

${ }^{\mathrm{a}} \mathrm{U}$, not detected at the indicated reporting limit.

${ }^{b}$ Quliafier $\mathrm{H}$ indicates that the holding time for the analysis was exceeded.

${ }^{c}$ Qualifier B indicates that the analyte was present in the blank.

${ }^{d}$ Qualifier E indicates that interference with the analysis was reported. 


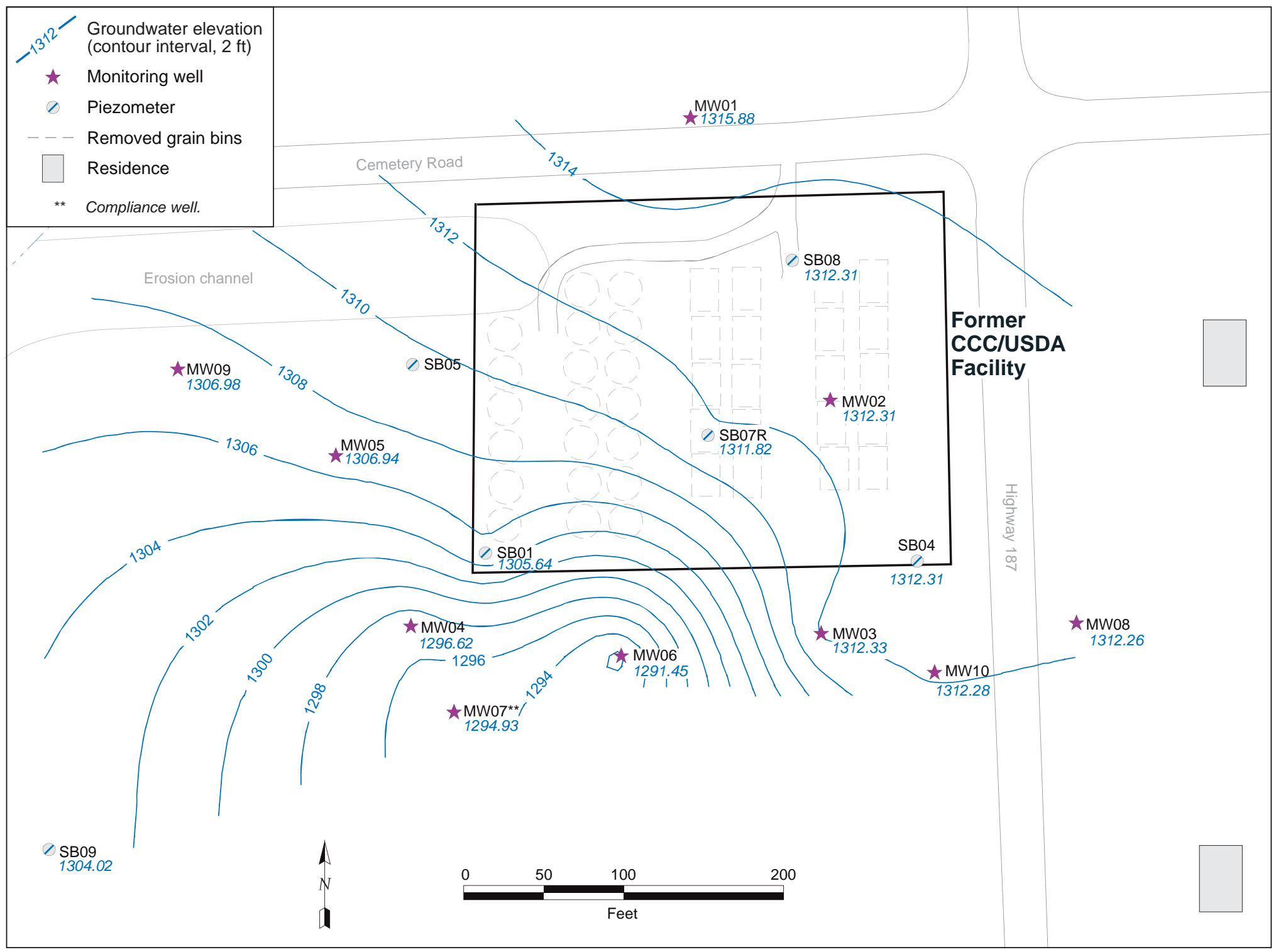

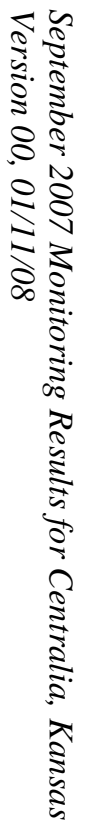

FIGURE 3.1 Potentiometric surface at Centralia, based on water levels measured manually on July 27, 2007. 


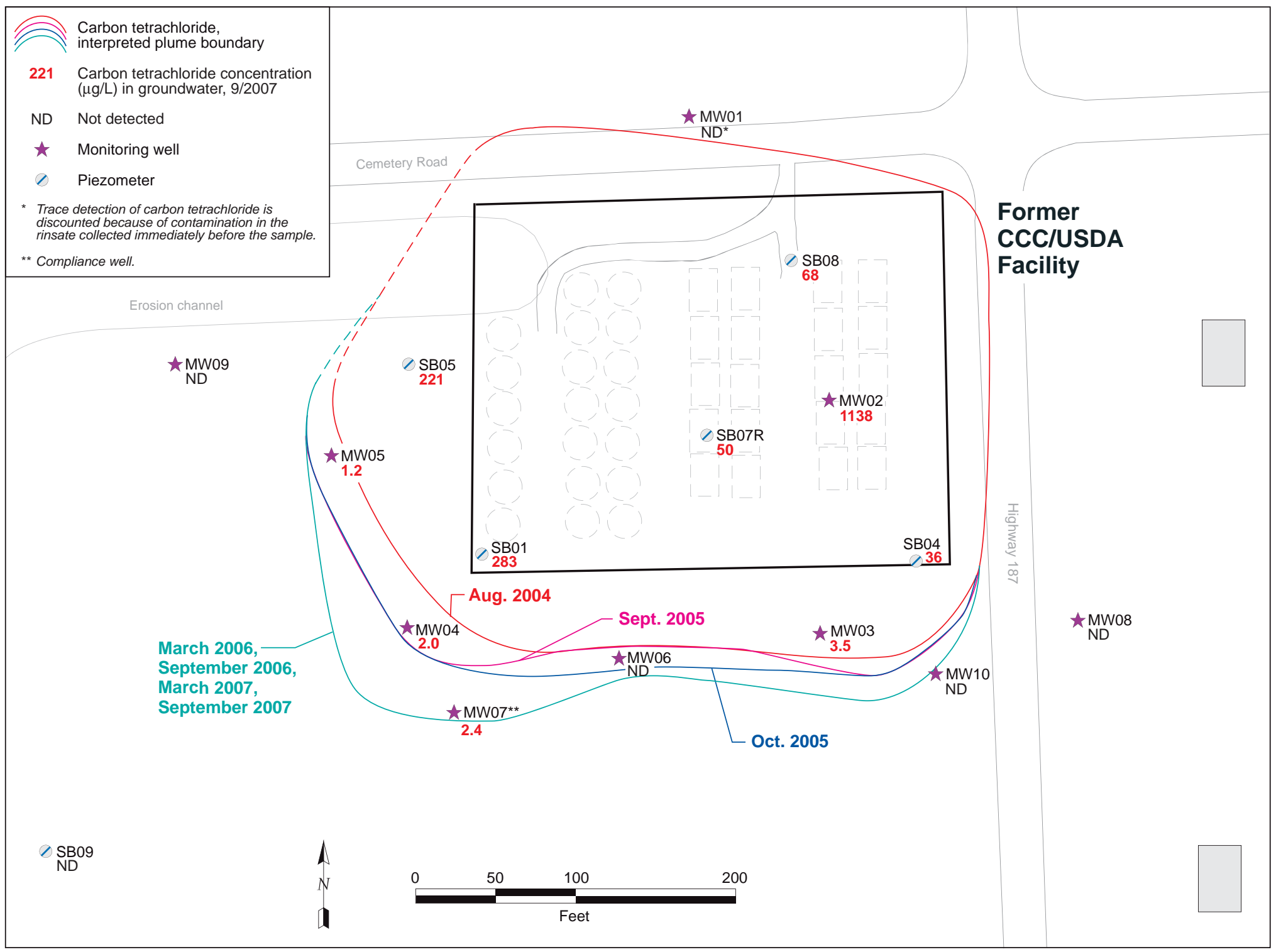

FIGURE 3.2 Carbon tetrachloride levels in groundwater at Centralia in March 2007, with the interpreted lateral extent of the contaminant at intervals during the period August 2004 to September 2007. 


\section{Conclusions and Recommendations}

\subsection{Conclusions}

The findings of the September 2007 monitoring event at Centralia support the following conclusions:

- Measurements of groundwater levels obtained manually and through the use of automatic recorders have consistently indicated an apparent direction of groundwater flow to the south-southwest across the former CCC/USDA facility.

- The September 2007 well sampling data are generally consistent with previous results. Longer-term trends of increasing carbon tetrachloride concentrations continue to be observed at monitoring points SB05, MW03, MW04, and MW07, along the western and southern margins of the groundwater plume. These trends suggest slow expansion of the plume at the downgradient locations.

- Sampling to date has yielded only limited evidence for the presence of subsurface conditions at Centralia conducive to anaerobic degradation of the carbon tetrachloride contamination in groundwater. Nevertheless, chloroform has consistently been detected at relatively elevated concentrations at monitoring points SB01, SB05, and (particularly) MW02, suggesting that carbon tetrachloride is being degraded at these locations.

\subsection{Recommendations}

The CCC/USDA developed an Interim Measure Conceptual Design (Argonne 2007b), proposing a pilot test of the Adventus EHC in situ chemical reduction (ISCR) technology, that was approved by the KDHE in November 2007 (KDHE 2007). Further information about the Adventus ISCR product is available online (http://www.adventusgroup.com/pdfs/Release-ISCRISCO.pdf). 
Groundwater sampling was incorporated into the design of the pilot test, to be implemented as part of the compliance monitoring program to evaluate the effectiveness of the EHC remediation technology at the Centralia site. Implementation of the proposed interim measure occurred in the late fall of 2007. The results presented here serve as pre-injection baseline data for existing monitoring points, per agreement with the KDHE. 


\section{References}

Argonne, 2002, Final Master Work Plan: Environmental Investigations at Former CCC/USDA Facilities in Kansas, 2002 Revision, ANL/ER/TR-02/004, prepared for the Commodity Credit Corporation, U.S. Department of Agriculture, Washington, D.C., by Argonne National Laboratory, Argonne, Illinois, December.

Argonne, 2003, Final Phase I Report and Phase II Work Plan: QuickSite ${ }^{\circledR}$ Investigation, Centralia, Kansas, ANL/ER/TR-02/009, prepared for the Commodity Credit Corporation, U.S. Department of Agriculture, Washington, D.C., by Argonne National Laboratory, Argonne, Illinois, March.

Argonne, 2004, Final Phase II Report: QuickSite ${ }^{\circledR}$ Investigation, Centralia, Kansas, ANL/ER/TR-03/006, prepared for the Commodity Credit Corporation, U.S. Department of Agriculture, Washington, D.C., by Argonne National Laboratory, Argonne, Illinois, March.

Argonne, 2005a, Final Report: 2004 Monitoring Well Installation and Sampling at Centralia, Kansas, ANL/ER/TR-04/011, prepared for the Commodity Credit Corporation, U.S. Department of Agriculture, Washington, D.C., by Argonne National Laboratory, Argonne, Illinois, October.

Argonne, 2005b, Final Work Plan: Groundwater Monitoring at Centralia, Kansas, ANL/ER/TR-05/004, prepared for the Commodity Credit Corporation, U.S. Department of Agriculture, Washington, D.C., by Argonne National Laboratory, Argonne, Illinois, August.

Argonne, 2006a, Final Report: Groundwater Monitoring at Centralia, Kansas, in SeptemberOctober 2005 and March 2006, with Expansion of the Monitoring Network in January 2006, ANL/EVS/AGEM/TR-06-06, prepared for the Commodity Credit Corporation, U.S. Department of Agriculture, Washington, D.C., by Argonne National Laboratory, Argonne, Illinois, October.

Argonne, 2006b, September 2006 Monitoring Results for Centralia, Kansas, ANL/EVS/AGEM/CHRON-1003, prepared for the Commodity Credit Corporation, U.S. Department of Agriculture, Washington, D.C., by Argonne National Laboratory, Argonne, Illinois, November 10. 
Argonne, 2007a, March 2007 Monitoring Results for Centralia, Kansas, ANL/EVS/AGEM/TR07-08, prepared for the Commodity Credit Corporation, U.S. Department of Agriculture, Washington, D.C., by Argonne National Laboratory, Argonne, Illinois, June.

Argonne, 2007b, Interim Measure Conceptual Design for Remediation at the Former CCC/USDA Grain Storage Facility at Centralia, Kansas: Pilot Test and Remedy Implementation, ANL/EVS/AGEM/TR-07-11, prepared for the Commodity Credit Corporation, U.S. Department of Agriculture, Washington, D.C., by Argonne National Laboratory, Argonne, Illinois, October.

EPA, 1995, Method 524.2: Measurement of Purgeable Organic Compounds in Water by Capillary Column Gas Chromatography/Mass Spectrometry, Revision 4.1, edited by J.W. Munch, National Exposure Research Laboratory, Office of Research and Development, U.S. Environmental Protection Agency, Cincinnati, Ohio.

EPA, 1998a, Test Methods for Evaluating Solid Waste: Physical/Chemical Methods, EPA SW846, 3rd edition, Draft Update IVA, U.S. Environmental Protection Agency, January (available from National Technical Information Service, 5285 Port Royal Road, Springfield, VA 22161).

EPA, 1998b, Technical Protocol for Evaluating Natural Attenuation of Chlorinated Solvents in Ground Water, EPA/600/R-98/128, Office of Research and Development, U.S. Environmental Protection Agency, Washington, D.C., September (http://www.epa.gov/superfund/resources/ gwdocs/protocol.htm).

Kampbell, D.H., and S.A. Vandegrift, 1998, "Analysis of Dissolved Methane, Ethane, and Ethylene in Ground Water by a Standard Chromatographic Technique," Journal of Chromatographic Science 36:253-256.

KDHE, 2001, Monitored Natural Attenuation, Policy BER-RS-042, Bureau of Environmental Remediation, Kansas Department of Health and Environment, Topeka, Kansas, March 30 (revised December 18, 2005; http://www.kdheks.gov/ber/policies/BER_RS_042.pdf).

KDHE, 2007, letter from C. Carey (Bureau of Environmental Remediation, Kansas Department of Health and Environment, Topeka, Kansas) to C. Roe (Commodity Credit Corporation, 
U.S. Department of Agriculture, Washington, D.C.), regarding review of Interim Measure Conceptual Design for Centralia, Kansas, November 9.

Sabetha, 2007, general receipt, Office of City Clerk, City of Sabetha, Kansas, acknowledging receipt of payment from Argonne National Laboratory for purged water, October 25. 


\section{Appendix A:}

Sequence of Sampling Activities at Centralia, Kansas, in September 2007 
TABLE A.1 Sequence of sampling activities at Centralia in September 2007.

\begin{tabular}{|c|c|c|c|c|c|c|c|c|}
\hline $\begin{array}{l}\text { Sample } \\
\text { Date }\end{array}$ & Time & Location & Sample & $\begin{array}{l}\text { Sample } \\
\text { Medium }\end{array}$ & Type $^{\mathrm{a}}$ & $\begin{array}{l}\text { Depth } \\
\text { (ft TOC) }\end{array}$ & $\begin{array}{c}\text { Chain of } \\
\text { Custody No. }\end{array}$ & Sample Description \\
\hline 09/24/07 & $11: 25$ & MW05 & CNMW05-W-16218 & Water & MW & $34.5-44.5$ & 6252 & $\begin{array}{l}\text { Depth to water from TOC }=10.80 \mathrm{ft} \text {. Depth of well from } \\
\text { TOC }=47.60 \mathrm{ft} \text {. Sample collected from } 4 \text {-in. monitoring } \\
\text { well at low flow after purging of } 72 \text { gal with Redi-Flo } \\
\text { pump. Purged to container. }\end{array}$ \\
\hline 09/24/07 & $12: 40$ & MW09 & CNMW09-W-16219 & Water & MW & $25-35$ & 6252 & $\begin{array}{l}\text { Depth to water from TOC }=4.00 \mathrm{ft} \text {. Depth of well from } \\
\text { TOC }=39.15 \mathrm{ft} \text {. Sample collected from } 2 \text {-in. monitoring } \\
\text { well at low flow after purging of } 18 \mathrm{gal} \text { with Redi-Flo } \\
\text { pump. Purged to container. }\end{array}$ \\
\hline 09/24/07 & $12: 41$ & MW09 & CNMW09D-W-16219 & Water & MW & $25-35$ & 6252 & Replicate of sample CNMW09-W-16219. \\
\hline 09/24/07 & $15: 42$ & MW07 & CNMW07-W-16221 & Water & MW & $45-55$ & 6252 & $\begin{array}{l}\text { Depth to water from TOC }=29.07 \mathrm{ft} \text {. Depth of well from } \\
\text { TOC }=58.70 \mathrm{ft} . \text { Sample collected from } 2 \text {-in. monitoring } \\
\text { well by using a valved tube after purging of } 15 \text { gal. } \\
\text { Purged to container. }\end{array}$ \\
\hline 09/24/07 & $16: 28$ & MW04 & CNMW04-W-16220 & Water & MW & $37.5-47.5$ & 6252 & $\begin{array}{l}\text { Depth to water from TOC }=26.24 \mathrm{ft} \text {. Depth of well from } \\
\text { TOC }=49.22 \mathrm{ft} . \text { Sample collected from } 4 \text {-in. monitoring } \\
\text { well at low flow after purging of } 40 \text { gal with Redi-Flo } \\
\text { pump. Purged to container. Well dry after } 25 \text { gal. } \\
\text { Sampled after recovery. }\end{array}$ \\
\hline 09/24/07 & $18: 01$ & MW06 & CNMW06-W-16222 & Water & MW & $46.5-56.5$ & 6252 & $\begin{array}{l}\text { Depth to water from TOC }=38.50 \mathrm{ft} \text {. Depth of well from } \\
\text { TOC }=60.00 \mathrm{ft} \text {. Sample collected from } 4 \text {-in. monitoring } \\
\text { well at low flow after purging of } 32 \text { gal with Redi-Flo } \\
\text { pump. Purged to container. Well dry at } 30 \text { gal. Sampled } \\
\text { after recovery. }\end{array}$ \\
\hline 09/25/07 & $8: 35$ & MW03 & CNMW03-W-16223 & Water & MW & $50.5-60.5$ & 6253 & $\begin{array}{l}\text { Depth to water from TOC }=22.60 \mathrm{ft} \text {. Depth of well from } \\
\text { TOC }=62.30 \mathrm{ft} \text {. Sample collected from } 4 \text {-in. monitoring } \\
\text { well at low flow after purging of } 75 \mathrm{gal} \text { with Redi-Flo } \\
\text { pump. Purged to container. }\end{array}$ \\
\hline 09/25/07 & $9: 52$ & MW10 & CNMW10-W-16224 & Water & MW & $30-45$ & 6253 & $\begin{array}{l}\text { Depth to water from TOC }=22.89 \mathrm{ft} \text {. Depth of well from } \\
\text { TOC }=47.71 \mathrm{ft} . \text { Sample collected from } 2 \text {-in. monitoring } \\
\text { well at low flow after purging of } 13 \mathrm{gal} \text { with Redi-Flo } \\
\text { pump. Purged to container. }\end{array}$ \\
\hline 09/25/07 & $11: 27$ & SB07R & CNSB07R-W-16225 & Water & $\mathrm{CPT} / \mathrm{P}$ & $45-60$ & 6253 & $\begin{array}{l}\text { Depth to water from TOC }=20.00 \mathrm{ft} \text {. Depth of well from } \\
\text { TOC }=58.50 \mathrm{ft} \text {. Sample collected from } 2 \text {-in. monitoring } \\
\text { well at low flow after purging of } 19 \text { gal with Redi-Flo } \\
\text { pump. Purged to container. }\end{array}$ \\
\hline
\end{tabular}


TABLE A.1 (Cont.)

\begin{tabular}{|c|c|c|c|c|c|c|c|c|}
\hline $\begin{array}{l}\text { Sample } \\
\text { Date }\end{array}$ & Time & Location & Sample & $\begin{array}{l}\text { Sample } \\
\text { Medium }\end{array}$ & Type & $\begin{array}{c}\text { Depth } \\
\text { (ft TOC) }\end{array}$ & $\begin{array}{c}\text { Chain of } \\
\text { Custody No. }\end{array}$ & Sample Description \\
\hline
\end{tabular}

\begin{tabular}{|c|c|c|c|c|c|c|c|c|}
\hline 09/25/07 & $13: 53$ & QC & CNQCTB-W-16234 & Water & TB & - & 6259 & $\begin{array}{l}\text { Trip blank sent to Envirosystems, Inc., for verification } \\
\text { organic analysis with samples listed on chain-of- } \\
\text { custody form (COC) } 6259 \text {. }\end{array}$ \\
\hline 09/25/07 & $13: 57$ & QC & CNQCTB-W-16235 & Water & TB & - & 6253 & $\begin{array}{l}\text { Trip blank sent to the AGEM Laboratory for organic } \\
\text { analysis with samples listed on COCs } 6252 \text { and } 6253 \text {. }\end{array}$ \\
\hline 09/25/07 & $13: 58$ & QC & CNQCFB-W-16236 & Water & FB & - & 6253 & $\begin{array}{l}\text { Field blank of water used during September } 2007 \text { sampling } \\
\text { event. }\end{array}$ \\
\hline 09/25/07 & $15: 52$ & MW08 & CNMW08-W-16226 & Water & MW & $38-53$ & 6253 & $\begin{array}{l}\text { Depth to water from TOC }=20.72 \mathrm{ft} \text {. Depth of well from } \\
\text { TOC }=57.10 \mathrm{ft} \text {. Sample collected from } 2 \text {-in. monitoring } \\
\text { well at low flow after purging of } 18 \text { gal with Redi-Flo } \\
\text { pump. Purged to container. }\end{array}$ \\
\hline 09/25/07 & $16: 30$ & QC & CNQCTB-W-16237 & Water & TB & - & 6263 & $\begin{array}{l}\text { Trip blank sent to Severn-Trent Laboratories for methane, } \\
\text { ethane, and ethene analyses with samples listed on } \\
\text { cOCs } 6254,6255,6260 \text {, and } 6263 \text {. }\end{array}$ \\
\hline 09/25/07 & $16: 32$ & QC & CNQCTB-W-16239 & Water & TB & - & 6255 & $\begin{array}{l}\text { Trip blank sent to Severn-Trent Laboratories for VOCs } \\
\text { analyses with samples listed on COCs } 6254,6255 \text {, } \\
6260 \text {, and } 6263 \text {. (Not required. Not analyzed.) }\end{array}$ \\
\hline 09/26/07 & $9: 24$ & MW02 & CNMW02-W-16227 & Water & MW & $49.5-59.5$ & 6265 & $\begin{array}{l}\text { Depth to water from TOC }=23.05 \mathrm{ft} \text {. Depth of well from } \\
\text { TOC }=61.25 \mathrm{ft} \text {. Sample collected from } 4 \text {-in. monitoring } \\
\text { well at low flow after purging of } 75 \mathrm{gal} \text { with Redi-Flo } \\
\text { pump. Purged to container. }\end{array}$ \\
\hline 09/26/07 & 9:25 & MW02 & CNMW02D-W-16227 & Water & MW & $49.5-59.5$ & 6265 & Replicate of sample CNMW02-W-16227. \\
\hline 09/26/07 & $10: 00$ & QC & CNQCRI-W-16240 & Water & $\mathrm{RI}$ & - & 6265 & $\begin{array}{l}\text { Rinsate of decontaminated sampling pump and hose after } \\
\text { collection of sample CNMW02-W-16227. }\end{array}$ \\
\hline 09/26/07 & $11: 15$ & MW01 & CNMW01-W-16228 & Water & MW & $54.5-64.5$ & 6265 & $\begin{array}{l}\text { Depth to water from TOC }=14.55 \mathrm{ft} \text {. Depth of well from } \\
\text { TOC }=69.50 \mathrm{ft} \text {. Sample collected from } 4 \text {-in. monitoring } \\
\text { well at low flow after purging of } 100 \mathrm{gal} \text { with Redi-Flo } \\
\text { pump. Purged to container. }\end{array}$ \\
\hline 09/26/07 & $11: 29$ & SB08 & CNSB08-W-16229 & Water & $\mathrm{CPT} / \mathrm{P}$ & $52-62$ & 6265 & $\begin{array}{l}\text { Depth to water from TOC }=21.37 \mathrm{ft} \text {. Depth of well from } \\
\text { TOC }=59.78 \mathrm{ft} \text {. Sample collected from } 1 \text {-in. monitoring } \\
\text { well by using a valved tube after purging of } 5 \text { gal. } \\
\text { Purged to container. }\end{array}$ \\
\hline 09/26/07 & 13:07 & SB04 & CNSB04-W-16230 & Water & $\mathrm{CPT} / \mathrm{P}$ & $51-61$ & 6265 & $\begin{array}{l}\text { Depth to water from TOC }=24.49 \mathrm{ft} \text {. Depth of well from } \\
\text { TOC }=59.37 \mathrm{ft} . \text { Sample collected from } 1 \text {-in. monitoring } \\
\text { well by using a valved tube after purging of } 5 \text { gal. } \\
\text { Purged to container. }\end{array}$ \\
\hline
\end{tabular}


TABLE A.1 (Cont.)

\begin{tabular}{|c|c|c|c|c|c|c|c|c|}
\hline $\begin{array}{l}\text { Sample } \\
\text { Date }\end{array}$ & Time & Location & Sample & $\begin{array}{l}\text { Sample } \\
\text { Medium }\end{array}$ & Type ${ }^{a}$ & $\begin{array}{l}\text { Depth } \\
\text { (ft TOC) }\end{array}$ & $\begin{array}{l}\text { Chain of } \\
\text { Custody No. }\end{array}$ & Sample Description \\
\hline 09/26/07 & $14: 06$ & SB09 & CNSB09-W-16231 & Water & СРТ/P & $32-42$ & 6265 & $\begin{array}{l}\text { Depth to water from TOC }=6.92 \mathrm{ft} \text {. Depth of well from } \\
\text { TOC }=35.85 \mathrm{ft} \text {. Sample collected from } 1 \text {-in. monitoring } \\
\text { well by using a valved tube after purging of } 3 \text { gal. } \\
\text { Purged to container. }\end{array}$ \\
\hline 09/26/07 & $15: 48$ & SB05 & CNSB05-W-16233 & Water & СРТ/P & $32-42$ & 6265 & $\begin{array}{l}\text { Depth to water from TOC }=11.75 \mathrm{ft} \text {. Depth of well from } \\
\text { TOC }=41.00 \mathrm{ft} . \text { Sample collected from } 1 \text {-in. monitoring } \\
\text { well by using a valved tube after purging of } 3 \text { gal. } \\
\text { Purged to container. }\end{array}$ \\
\hline 09/27/07 & $7: 22$ & SB01 & CNSB01-W-16232 & Water & СРТ/P & $40-50$ & 6269 & $\begin{array}{l}\text { Depth to water from TOC }=20.05 \mathrm{ft} \text {. Depth of well from } \\
\text { TOC }=49.00 \mathrm{ft} \text {. Sample collected on Sept. } 27 \text { from } 1 \text {-in. } \\
\text { monitoring well by using a valved tube. Well was dry } \\
\text { after purging of } 1.5 \mathrm{gal} \text { on Sept } 26 \text {. Purged to container. }\end{array}$ \\
\hline 09/27/07 & $7: 57$ & QC & CNQCTB-W-16244 & Water & TB & - & 6269 & $\begin{array}{l}\text { Trip blank sent to the AGEM Laboratory for organic } \\
\text { analyses with samples listed on COCs } 6265 \text { and } 6269 \text {. }\end{array}$ \\
\hline 09/27/07 & $12: 30$ & QC & CNQCTB-W-16242 & Water & TB & - & 6268 & $\begin{array}{l}\text { Trip blank sent to Severn-Trent Laboratories for methane, } \\
\text { ethane, and ethene analyses with samples listed on } \\
\text { COCs } 6267,6268,6273 \text {, and } 6274 \text {. }\end{array}$ \\
\hline 09/27/07 & $12: 35$ & QC & CNQCTB-W-16243 & Water & TB & - & 6274 & $\begin{array}{l}\text { Trip blank sent to Severn-Trent Laboratories for VOCs } \\
\text { analyses with samples listed on COCs } 6267,6268, \\
6273 \text {, and } 6274 \text {. (Not required. Not analyzed.) }\end{array}$ \\
\hline $10 / 09 / 07$ & $6: 00$ & QC & CN-WW & Water & BT & - & 1132596 & $\begin{array}{l}\text { Containerized waste purge water generated during } \\
\text { September } 2007 \text { monitoring event. Analyzed at Pace } \\
\text { Analytical Services for VOCs, ethylene dibromide, and } \\
\text { nitrate. }\end{array}$ \\
\hline
\end{tabular}

a Sample types: BT, wastewater composite; CPT/P, cone penetrometer piezometer; FB, field blank; RI, rinsate; MW, monitoring well; TB, trip blank. 


\section{Appendix B:}

Data Summary for Verification VOCs analyses by Envirosystems, Inc. 


\section{ENVIROSYSTEMS, INC.}

9200 Rumsey Road - Suite B102 - Columbia, Maryland 21045-1934

Phone (410) 964-0330 - Fax (410) 740-9306

Email: info@envsystems.com •Webpage: www.envsystems.com/envsys

October 10, 2007

Jorge S. Alvarado, PH. D

Argonne National Laboratory

Environmental Research Division

Applied Geosciences and Environmental

Management Section

9700 South Cass Avenue, ER-203

Argonne, Illinois 60439

RE: Report \#070287

Dear Jorge,

Enclosed is the Analytical Data Package for Organics Analysis for the samples received on September 26,2007. These samples were analyzed by using method SW-846 8260B and USEPA CLP SOWOLM04.3 and the chain of custody instructions.

Please do not hesitate to call if you have any questions, comments, or require additional information.

Sincerely,

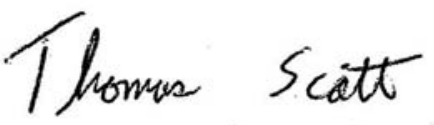

Mohan Khare Ph.D.

President/CEO

Enclosure (1)

$\mathrm{MK} / \mathrm{ncc}$

Envirosystems, Inc.

Report R070287 


\section{SDG NARRATIVE \\ VOLATILE ORGANICS (VOC)}

Envirosystems, Inc.

Contract: N/A

Client: Argonne National Laboratory

Case: N/A

SDG: ARG70912

\section{SAMPLE RECIEPT}

Date received: 09-26-2007

Cooler Temperature: 2

Sample Summary

\begin{tabular}{|c|c|c|c|}
\hline Client ID & Laboratory ID & Matrix & pH \\
\hline CN-MW03-W-16223 & $0070912-01$ & WATER & 7 \\
\hline CN-SB07R-W-16225 & $0070912-02$ & WATER & 7 \\
\hline CN-QCTB-W-16234 & $0070912-03$ & WATER & 7 \\
\hline
\end{tabular}

\section{HOLDING TIMES}

A. Sample Preparation: All holding times were met.

B. Sample Analysis: All holding times were met.

\section{METHODS}

The samples were analyzed and reported by using method SW-846 8260B and USEPA CLP SOW OLM04.3 for target compound list.

\section{INSTRUMENT AND CHROMATOGRAPHIC CONDITIONS}

A Hewlett Packard 6890 gas chromatograph equipped with a Hewlett Packard 5975 MSD was used fc sample analysis. The capillary column used was a Restek $20 \mathrm{~m}$ by $0.18 \mathrm{~mm} \mathrm{ID} \mathrm{by} 1.0 \mu \mathrm{m}$ film thickn (Restek Cat. \# RTX-624). The trap used with the sample concentrator is an OI Analytical Trap \#10, 3 packed with Tenax/silica gel/cms (PN\#228122).

\section{PREPARATION}

The submitted samples were analyzed as received.

\section{ANALYSIS}

\section{A. Calibration:}

\section{Initial calibration}

All acceptance criteria as stipulated by SW- $8468260 \mathrm{~b}$ were met for all SPCC's and CCC's. All target compounds met the required percent RSD. 


\section{SDG NARRATIVE VOLATILE ORGANICS (VOC)}

\section{Blanks:}

All acceptance criteria were met.

\section{Surrogates:}

All acceptance criteria were met except for sample CN-MW03-W-16223, two surrogates were outside qc limits.

B. Spikes:

I. Laboratory Control Spikes (LCS)

LCS and LCSD samples were not analyzed.

\section{Matrix Spike/Matrix Spike Duplicate (MS/MSD)}

The client did not request a MS/MSD.

\section{Internal Standards:}

All acceptance criteria were met.

\section{Samples}

Sample analysis proceeded normally.

I certify that this Sample Data Package is in compliance with the terms and conditions of the contract, both technically and for completeness, for other than the conditions detailed above. Release of the data contained in the hard copy Sample Data Package and in the Electronic Data Deliverables has been authorized by the laboratory manager or the manager's designee, as verified by the following signatures.

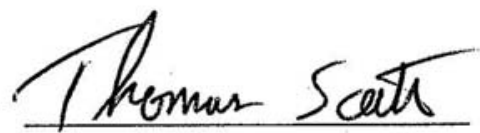

Laboratory Manager

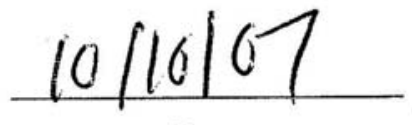

Date 


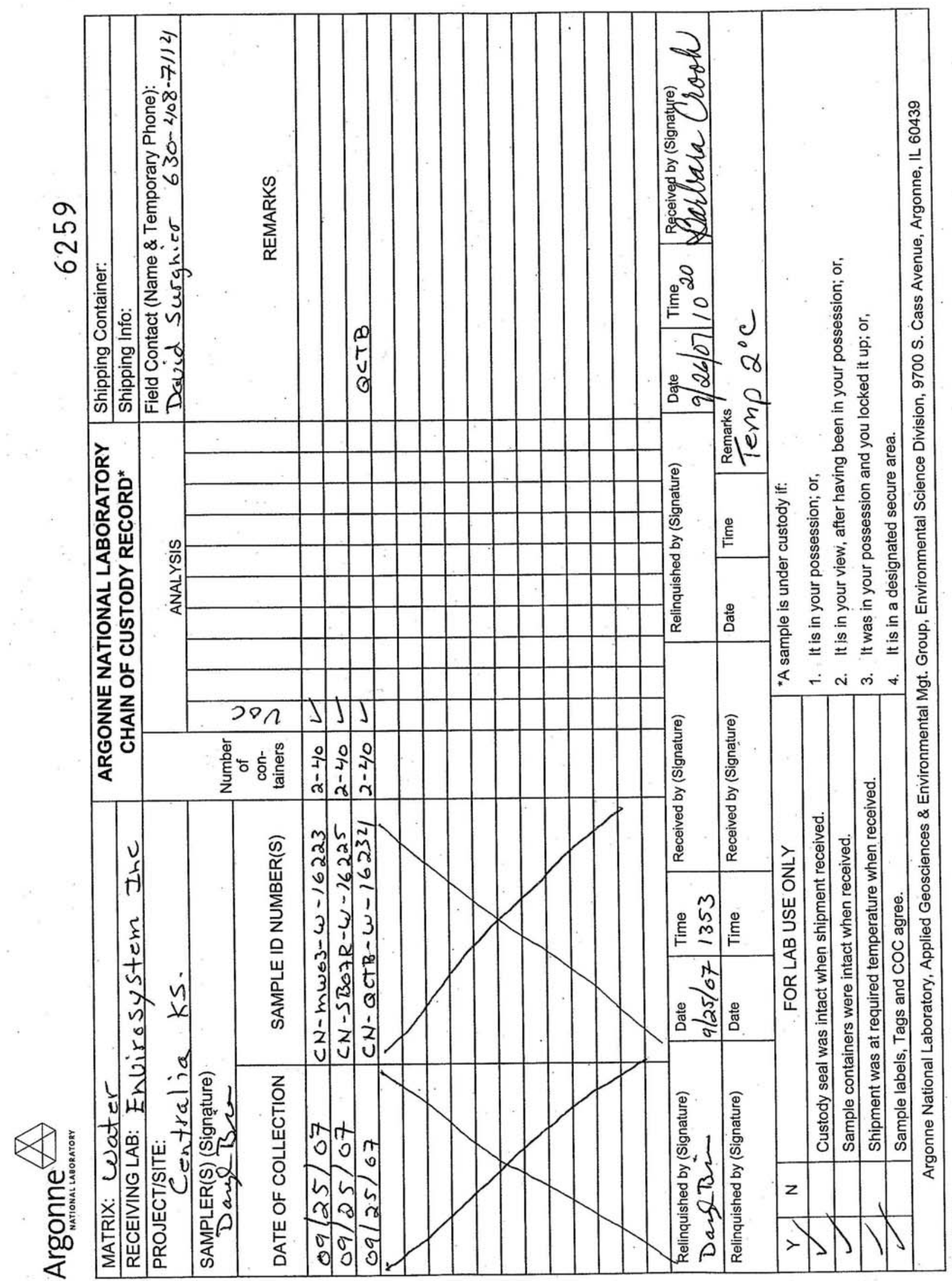


$1 A$ - FORM I VOA-1

VOLATILE ORGANICS ANALYSIS DATA SHEET
EPA SAMPLE NO.

CN-MW03-W-16223
Lab Name: Envirosystems, Inc.

Lab Code: ENVSYS Case No.:

Matrix: (SOIL/SED/WATER) WATER

Sample wt/vol: $5.00(\mathrm{~g} / \mathrm{mL}) \mathrm{ML}$

Level: (TRACE/LOW/MED)

LOW

\% Moisture: not dec.

GC Column: $\quad$ RTX-624 ID: 0.18 (mm)

Soil Extract Volume:

Purge Volume:

$$
5.00
$$

Contract:

Mod. Ref No.: SDG No.: $\quad$ Arg70912 Lab Sample ID: 0070912-01 Lab File ID: H73FA058. D

Date Received:

$09 / 26 / 2007$

Date Analyzed:

$10 / 02 / 2007$

Dilution Factor:

1.0

Soil Aliquot Volume:

(uL)

\begin{tabular}{|l|l|c|c|}
\hline CAS NO: & COMPOUND & $\begin{array}{c}\text { CONCENTRATION UNITS: } \\
\text { (ug/L or ug/kg) UG/L }\end{array}$ & Q \\
\hline $75-71-8$ & Dichlorodifluoromethane & 5.0 & $\mathrm{U}$ \\
\hline $74-87-3$ & Chloromethane & 5.0 & $\mathrm{U}$ \\
\hline $75-01-4$ & Vinyl chloride & 5.0 & $\mathrm{U}$ \\
\hline $74-83-9$ & Bromomethane & 5.0 & $\mathrm{U}$ \\
\hline $75-00-3$ & Chloroethane & 5.0 & $\mathrm{U}$ \\
\hline $75-69-4$ & Trichlorofluoromethane & 5.0 & $\mathrm{U}$ \\
\hline $75-35-4$ & $1,1-$ Dichloroethene & 5.0 & $\mathrm{U}$ \\
\hline $76-13-1$ & $1,1,2-T r i c h l o r o-1,2,2-$ trifluoroethane & $\mathrm{U}$ \\
\hline $67-64-1$ & Acetone & 5.0 & $\mathrm{U}$ \\
\hline $75-15-0$ & Carbon disulfide & 5.0 & $\mathrm{U}$ \\
\hline $79-20-9$ & Methyl acetate & 5.0 & $\mathrm{U}$ \\
\hline $75-09-2$ & Methylene chloride & 5.0 & $\mathrm{U}$ \\
\hline $156-60-5$ & trans-1,2-Dichloroethene & 5.0 & $\mathrm{U}$ \\
\hline $1634-04-4$ & Methyl tert-butyl ether & 5.0 & $\mathrm{U}$ \\
\hline $75-34-3$ & $1,1-D i c h l o r o e t h a n e$ & 5.0 & $\mathrm{U}$ \\
\hline $156-59-2$ & Cis-1,2-Dichloroethene & 5.0 & $\mathrm{U}$ \\
\hline $78-93-3$ & 2-Butanone & 5.0 & $\mathrm{U}$ \\
\hline $67-66-3$ & Chloroform & 5.0 & $\mathrm{U}$ \\
\hline $71-55-6$ & $1,1,1-$ Trichloroethane & 5.0 & $\mathrm{U}$ \\
\hline $110-82-7$ & Cyclohexane & 5.0 & $\mathrm{U}$ \\
\hline $56-23-5$ & Carbon Tetrachloride & 2.6 & $\mathrm{~J}$ \\
\hline $71-43-2$ & Benzene & 5.0 & $\mathrm{U}$ \\
\hline $107-06-2$ & $1,2-D i c h l o r o e t h a n e$ & 5.0 & $\mathrm{U}$ \\
\hline
\end{tabular}


$1 B$ - FORM I VOA-2

VOLATILE ORGANICS ANALYSIS DATA SHEET
EPA SAMPLE NO.

CN-MW03-W-16223

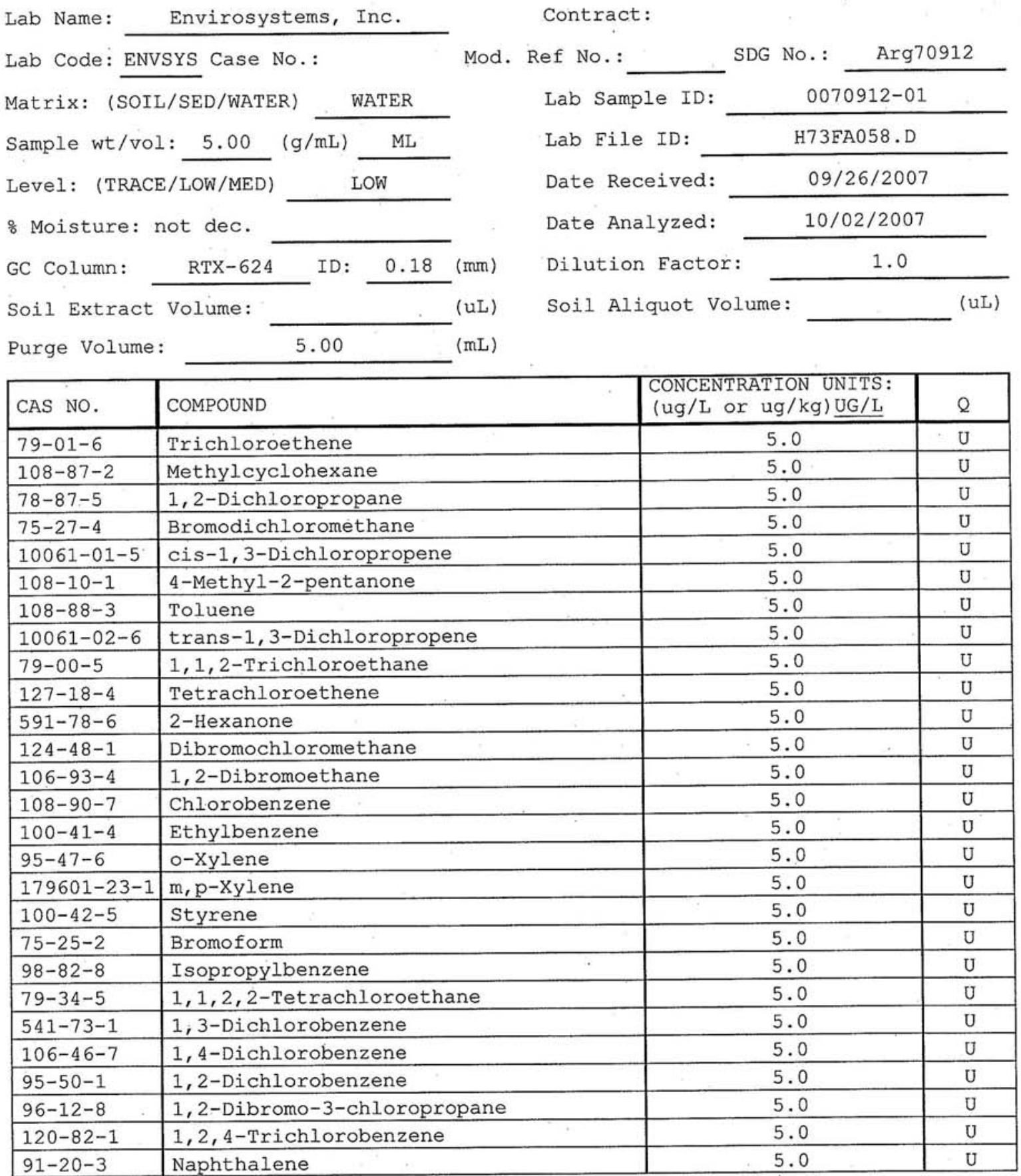


$1 A$ - FORM I VOA-1

VOLATILE ORGANICS ANALYSIS DATA SHEET
EPA SAMPLE NO.

CN-SB07R-W-16225

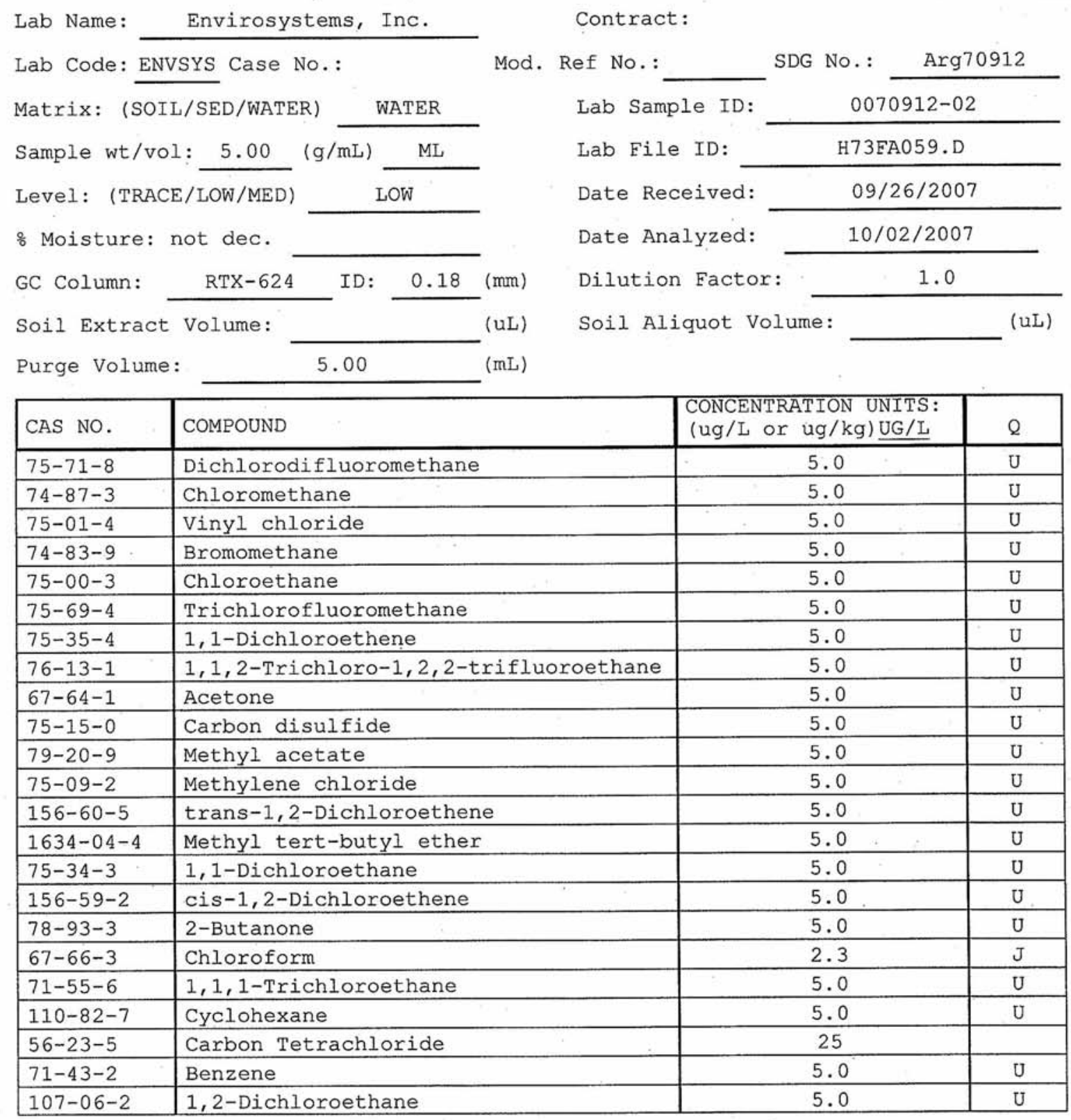


$1 B$ - FORM I VOA-2

VOLATILE ORGANICS ANALYSIS DATA SHEET
EPA SAMPLE NO.

CN-SB07R-W-16225

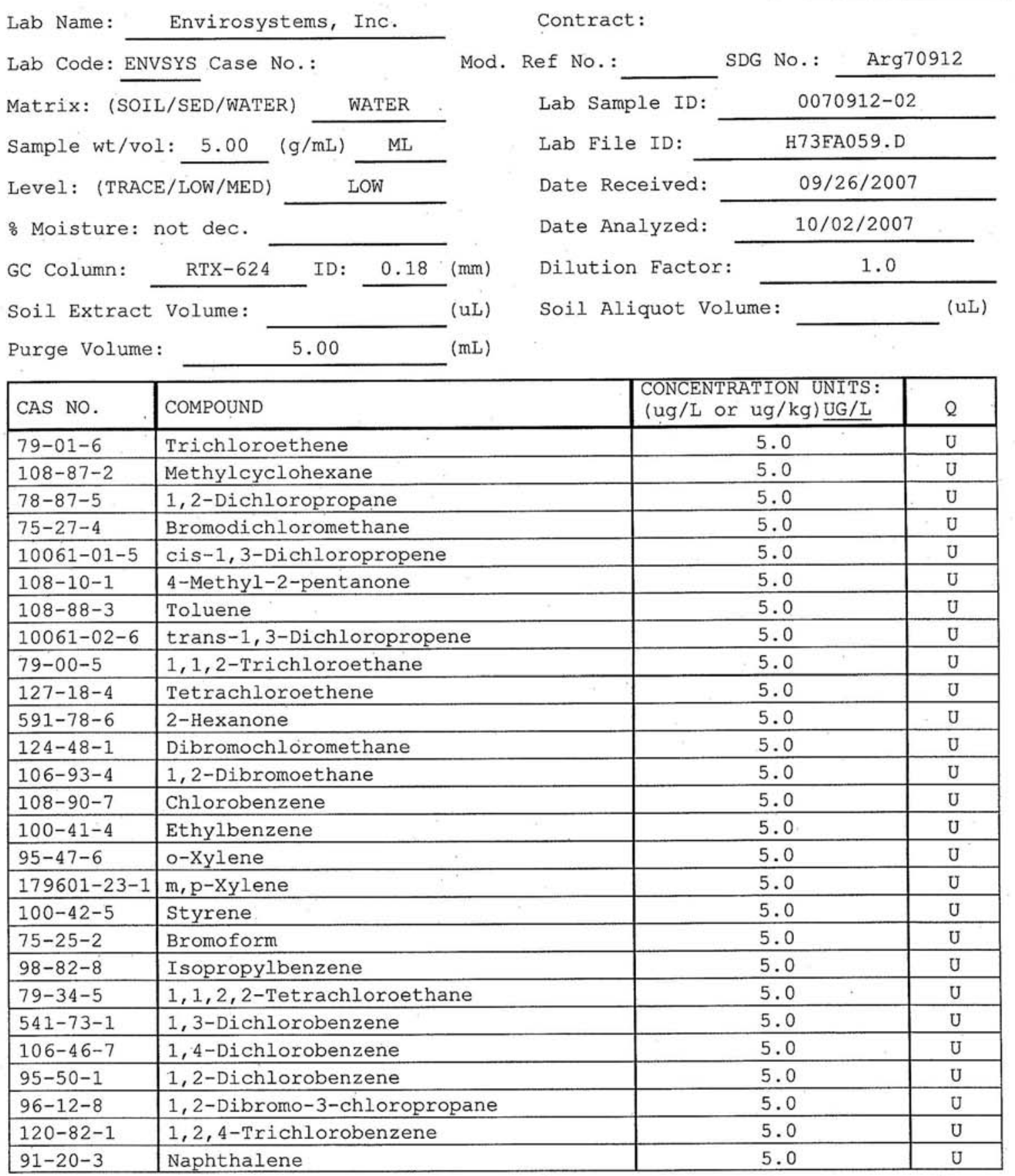


$1 A$ - FORM I VOA-1

VOLATILE ORGANICS ANALYSIS DATA SHEET
EPA SAMPLE NO.

CN-QCTB-W-16234

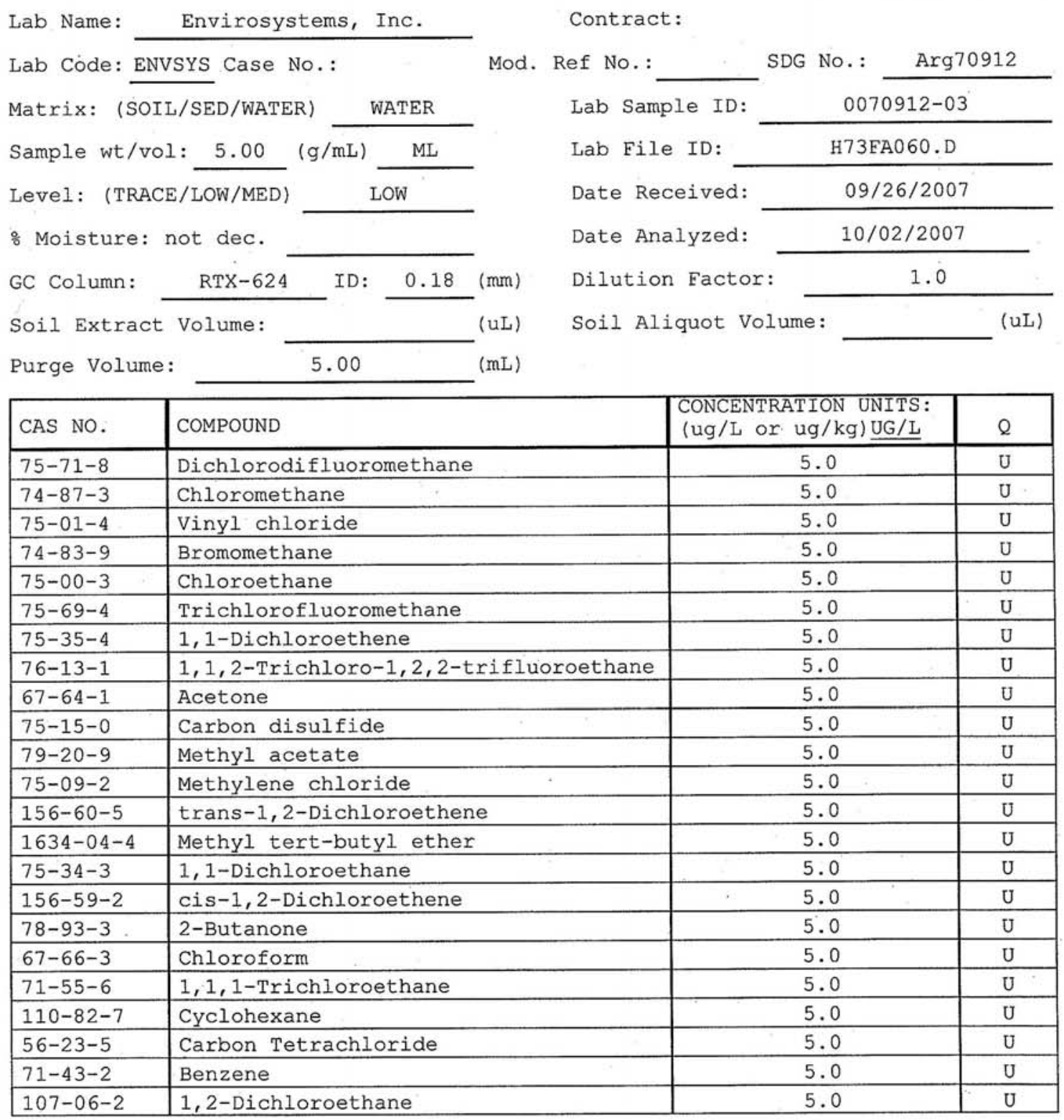


$1 B$ - FORM I VOA-2

VOLATILE ORGANICS ANALYSIS DATA SHEET
EPA SAMPLE NO.

CN-QCTB-W-16234

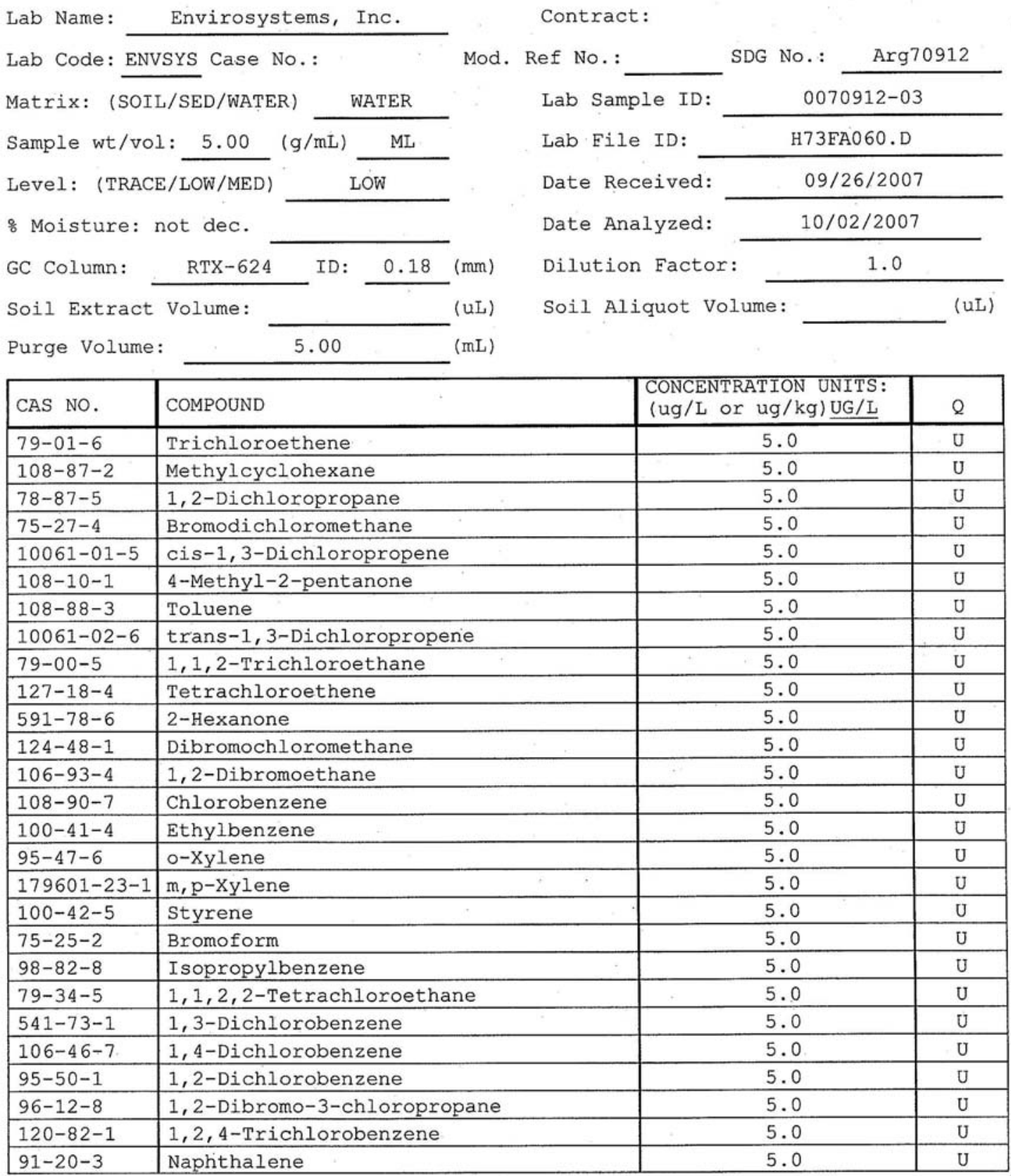


Argonne

Environmental Science Division

Argonne National Laboratory

9700 South Cass Avenue, Bldg. 203

Argonne, IL 60439-4843

www.anl.gov

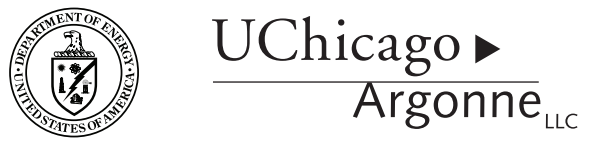

A U.S. Department of Energy laboratory

managed by UChicago Argonne, LLC 